University of Wollongong

Research Online

Faculty of Engineering and Information

Faculty of Engineering and Information

Sciences - Papers: Part A

Sciences

$1-1-2015$

\title{
Nonlinear bilateral teleoperation using extended active observer for force estimation and disturbance suppression
}

\author{
Linping Chan \\ University of Wollongong, Ic842@uowmail.edu.au \\ Fazel Naghdy \\ University of Wollongong, fazel@uow.edu.au \\ David Stirling \\ University of Wollongong, stirling@uow.edu.au \\ Matthew Field \\ University of Wollongong, field@uow.edu.au
}

Follow this and additional works at: https://ro.uow.edu.au/eispapers

Part of the Engineering Commons, and the Science and Technology Studies Commons

Research Online is the open access institutional repository for the University of Wollongong. For further information contact the UOW Library: research-pubs@uow.edu.au 


\title{
Nonlinear bilateral teleoperation using extended active observer for force estimation and disturbance suppression
}

\author{
Abstract \\ A novel nonlinear teleoperation algorithm for simultaneous inertial parameters and force estimation at \\ the master and slave sides of the teleoperation system is proposed. The scheme, called Extended Active \\ Observer (EAOB), is an extension of the existing active observer. It provides effective force tracking at the \\ master side with accurate position tracking at the slave side in the presence of inertial parameter \\ variation and measurement noise. The proposed method only requires the measurement of robot \\ position, and as a result significantly reduces the difficulty and cost of implementing bilateral \\ teleoperation systems. The approach is described and its stability is analytically verified. The \\ performance of the method is validated through computer simulation and compared with the Nicosia \\ observer-based controller. According to the results, EAOB outperforms the Nicosia observer method and \\ effectively rejects noise.

\section{Keywords} \\ Teleoperation, Adaptive control, Extended active observer, Force estimation, Disturbance rejection \\ Disciplines \\ Engineering | Science and Technology Studies

\section{Publication Details} \\ L. Chan, F. Naghdy, D. A. Stirling \& M. Field, "Nonlinear bilateral teleoperation using extended active \\ observer for force estimation and disturbance suppression," Robotica, vol. 33, (1) pp. 61-86, 2014.
}




\title{
Nonlinear bilateral teleoperation using extended active observer for force estimation and disturbance suppression
}

Linping Chan*, Fazel Naghdy, David Stirling, Matthew Field

School of Electrical, Computer \& Telecommunications Engineering, University of Wollongong, Wollongong NSW 2522 Australia

\begin{abstract}
A novel nonlinear teleoperation algorithm for simultaneous inertial parameters and force estimation at both master and slave sides of the teleoperation system is proposed. The scheme, called Extended Active Observer (EAOB), is an extension of the existing active observer. It provides effective force tracking at the master side with accurate position tracking at the slave side in the presence of inertial parameter variation and measurement noise. The proposed method only requires the measurement of robot position and as a result significantly reduces the difficulty and cost of implementing bilateral teleoperation systems. The approach is described and its stability is analytically verified. The performance of the method is validated through computer simulation and compared with Nicosia observer based controller. According to the results, EAOB outperforms Nicosia-observer method and effectively rejects noise.
\end{abstract}

Key words: Teleoperation; Adaptive control; Extended active observer; Force estimation; Disturbance rejection

\section{Introduction}

In general, a classical bilateral teleoperation system consists of five interconnected elements: a human operator that issues commands through a haptic interface or master device controlling a remotely located teleoperator interacting with its surrounding environment via communication channels connecting the master device and local teleoperator. Since different types of command and feedback signals can be exchanged between the master and slave manipulators, a number of bilateral teleoperation architectures may emerge: position-position, position-force, force-position, and force-force architectures. Among these four teleoperation architectures, two common bilateral teleoperation approaches are position-position and position-force architectures $1 .{ }^{1}$ In the position-position architecture, the master position is passed to the slave side, and the slave position is fed back to the master side. This approach does not measure the force and merely attempts to minimize the difference 
between the master and slave position, and feed a force proportional to the difference back to the user once the slave makes contact with an object. This kind of approach could not achieve ideal force tracking. This approach suffers from a distorted perception in free-motion condition and cannot produce ideal force tracking. Hence, the absence of a slave-side force sensor, results in control inaccuracies produced by nonzero position errors, leading to proportional force feedback to the user even when the slave is not in contact with the environment.

On the contrary, the position-force architecture requires a force sensor to measure the interactions between the slave and the environment, and directly transmitting the force signal to the master side for control. Compared to the position-position architecture, perfect force tracking can be attained in this approach. Furthermore, the distorted perception in free-motion condition is largely decreased by using position-force architecture. ${ }^{2}$ The increased performance, from a transparency point of view, of the position-force architecture motivates research into teleoperation systems where force is measured. Force sensors, however, are not always possible in practice. In some teleoperation applications such as minimal invasive surgery, it is difficult to attach force and torque sensors at the end of laparoscopic surgical devices. Furthermore, it is well-known that force and torque sensors amplify noise, which will result in noise control signals. As a result, using force and torque sensors is not realistic and desirable.

To overcome such challenges, force observers have been gradually deployed in teleoperation systems to estimate forces instead of using force sensors. Chen et al. ${ }^{3}$ propose a nonlinear disturbance observer for force estimation based on the idea of modifying estimation by the difference between the estimated output and the actual output. Daly et al. ${ }^{4}$ apply sliding mode observer to a teleoperation system to estimate the force rendered by the human operator and environment based on position. Ahn et al. ${ }^{5}$ deploy Nicosia observer ${ }^{6}$ to evaluate reaction forces produced through the interaction between human and environment in a teleoperation system. However, the majority of the force observers reported in the literature can only effectively estimate the force rendered by the environment in the absence of disturbance and measurement noise. Practical robotic systems are subject to different types of disturbances, such as inertial parameter variations, frictions, and noises, that, when unaccounted for, can cause poor force estimation in the observers.

In this work, we address this gap in design of teleoperation systems and propose an Extended Active Observer (EAOB) that can simultaneously handle the problem of external force estimation and disturbance suppression. Through the combination of the EAOB and classical position-force teleoperation architecture, a novel positionforce architecture with hardware requirements of a position-position architecture that provides the benefits of a 
classical position-force architecture is developed. In this architecture, input signals of the master and slave controller and all transmitted signals between the master and slave manipulators are obtained by the EAOB. Furthermore, the performance of EAOB based control scheme is compared against Nicosia observer based control scheme for a typical 2 DOF nonlinear teleoperation system. The results show that the EAOB outperforms Nicosia observer for a time variant nonlinear teleoperation system, and demonstrates better noise rejection properties. Most noticeably, the proposed approach offers the following contributions compared to the previous work:

1) This work proposes an EAOB based teleoperation scheme, which can achieve both accurate force tracking at the master side and position tracking at the slave side by using the estimated position, velocity, and force signals. This design significantly reduces the cost of the entire teleoperation system.

2) The proposed EAOB for force estimation can be applied to time variant dynamic systems. In EAOB, we assume a time variant model for both the master and slave manipulators and update their model parameters in real time to ensure accurate operator/environment rendered force estimation. This makes the proposed observer more reliable and effective in real world applications compared to current force estimators that do not consider inertial robot parameter variations during the estimation process.

3) Given the imperfect measurement process in real world applications, the proposed EAOB also takes into account the effect of measurement noise on force estimation and system performance, and attains a better force estimation and accurate force and position tracking at the master and slave sides, respectively. This is a major characteristic of the EAOB compared to other force estimators that cannot function well in the presence of measurement noise.

4) The proposed EAOB deploys the theoretical concepts behind Kalman filter to optimally design the observer gain. This is also another advantage of our method compared to other force observers in which the adaptive gains are determined through trial and error.

The remaining part of the paper is organized as follows: The dynamic model of a teleoperation system will be studied in Section 2. This will be followed by presenting the proposed EAOB based position-force control architecture for the teleoperation system in Section 3. Application of EAOB to a 2 DOF nonlinear teleoperation system through computer simulation is presented in Section 4. Finally, a brief conclusion will be drawn in Section 5 and some future research directions will be outlined.

\section{Dynamics of a teleoperation system}


Generally, a teleoperation system can be viewed as a pair of robotic subsystems known as master and slave manipulators, modelled as a couple of n-Degrees of Freedom (DOF) serial links. Taking into account the torques exerted by the human operator and environment on the master and slave manipulators, the nonlinear dynamical model of a teleoperation system in the joint space can be formulated as

$$
\begin{gathered}
M_{m}\left(q_{m}, \theta_{m}\right) \ddot{q}_{m}+V_{m}\left(q_{m}, \dot{q}_{m}, \theta_{m}\right) \dot{q}_{m}+g_{m}\left(q_{m}, \theta_{m}\right)=T_{m}+T_{h}, \\
M_{s}\left(q_{s}, \theta_{s}\right) \ddot{q}_{s}+V_{s}\left(q_{s}, \dot{q}_{s}, \theta_{s}\right) \dot{q}_{s}+g_{s}\left(q_{s}, \theta_{s}\right)=T_{s}-T_{e},
\end{gathered}
$$

where $\ddot{q}_{*}, \dot{q}_{*}, q_{*}\left(*=m\right.$ or $s$ ) are angular acceleration, angular velocity and angular position signals, $M_{*}\left(q_{*}, \theta_{*}\right)$ is the inertia matrix, $V_{*}\left(q_{*}, \dot{q}_{*}, \theta_{*}\right)$ is the vector of Coriolis and centripetal terms, $g_{*}\left(q_{*}, \theta_{*}\right)$ is the gravity torque, $T_{*}$ are input torques of the controllers, $\theta_{*}$ represent inertial robotic parameters, and $T_{h}, T_{e}$ correspond to the torques exerted by the human operator and environment, respectively.

Given that the control design is preferred using the acceleration, velocity, position, and force signals in the robot task space, the dynamic model in (1) will be further explored. The relationship between the end effector coordinates and joint angles can be expressed through forward kinematics. Meanwhile, the reaction torques $T_{h}$, and $T_{e}$ from the operator and environment are also related to the reaction force $f_{h}, f_{e}$ at the end effector through the master and slave manipulator Jacobian, respectively. That is,

$$
\begin{aligned}
& T_{h}=J_{m}^{T}\left(q_{m}\right) f_{h}, \\
& T_{e}=J_{s}^{T}\left(q_{s}\right) f_{e},
\end{aligned}
$$

where $J_{*}\left(q_{*}\right),(*=m / s)$ is the manipulator Jacobian matrix.

Thus, the dynamics of the system (1) can be expressed in term of acceleration in state space form as

$$
\begin{aligned}
& {\left[\begin{array}{l}
\dot{q}_{m} \\
\ddot{q}_{m}
\end{array}\right]=\left[\begin{array}{c}
\dot{q}_{m} \\
M_{m}^{-1}\left(q_{m}, \theta_{m}\right)\left(-V_{m}\left(q_{m}, \dot{q}_{m}, \theta_{m}\right) \dot{q}_{m}-g_{m}\left(q_{m}, \theta_{m}\right)+T_{m}+J_{m}^{T}\left(q_{m}\right) f_{h}\right)
\end{array}\right] .} \\
& {\left[\begin{array}{c}
\dot{q}_{s} \\
\ddot{q}_{s}
\end{array}\right]=\left[\begin{array}{c}
\dot{q}_{s} \\
M_{s}^{-1}\left(q_{s}, \theta_{s}\right)\left(-V_{s}\left(q_{s}, \dot{q}_{s}, \theta_{s}\right) \dot{q}_{s}-g_{s}\left(q_{s}, \theta_{s}\right)+T_{s}-J_{s}^{T}\left(q_{s}\right) f_{e}\right)
\end{array}\right] .}
\end{aligned}
$$

With the Computed Torque Method (CTM), the master and slave controllers are designed as

$$
T_{m}=M_{m}\left(q_{m}, \theta_{m}\right) \ddot{q}_{m}+V_{m}\left(q_{m}, \dot{q}_{m}, \theta_{m}\right) \dot{q}_{m}+g_{m}\left(q_{m}, \theta_{m}\right)-J_{m}^{T}\left(q_{m}\right) f_{h} .
$$




$$
T_{s}=M_{s}\left(q_{s}, \theta_{s}\right) \ddot{q}_{s}+V_{s}\left(q_{s}, \dot{q}_{s}, \theta_{s}\right) \dot{q}_{s}+g_{s}\left(q_{s}, \theta_{s}\right)+J_{s}^{T}\left(q_{s}\right) f_{e} .
$$

The performance of the controller $T_{*}(*=m / s)$ depends highly on the accuracy of position and velocity $q_{*}, \dot{q}_{*}$, inertial robot manipulator parameter $\theta_{*}$, and external force $f_{h}, f_{e}$. Due to measurement noise, feeding back of position $q_{*}$, velocity $\dot{q}_{*}$, and external forces $f_{h}, f_{e}$, to the master or slave controllers will result in noisy control signals that in turn will destabilize the system. As a consequence, developing observers to estimate these signals is an effective way to avoid these challenges. Due to inevitable parameter variation of the system, an update law is also required to estimate inertial robot manipulator parameters $\theta_{*}$. An extended Kalman filter in ${ }^{7}$ is deployed to update these parameters in real time.

\section{EAOB-based control of teleoperation systems}

In this Section, the position-force teleoperation architecture for a nonlinear teleoperation system is presented first. Then, the EAOB proposed to estimate the external forces on the master and slave sides is introduced, and incorporated into the position-force architecture of a nonlinear teleoperation system. Finally, control laws for the master and slave sides are developed, and stability of the entire teleoperation system is analyzed.

\subsection{The position-force teleoperation architecture}

Force feedback is a key element in bilateral teleoperation. It is what allows the operator to have a sense of presence at the remote environment. The force feedback assists operator to manipulate the remote environment more effectively. For example, in remote surgery, a surgeon can apply excessive force with the surgical tools and potentially injure a patient ${ }^{8}$. Force feedback traditionally requires force sensors that in turn can add to the cost and complexity of the process by introducing measurement noise ${ }^{9}$. Meanwhile, inertial robot parameter variations in the teleoperation system often cause the obtained forces inaccurate. In order to overcome such challenges, a novel position-force architecture based control approach, achieved by using the estimated signals in communication channels and controller design, is proposed in this work, as illustrated in Fig. 1.

In this architecture, the inputs of the master and slave controllers and all the transmitted signals between the master and slave manipulators are obtained by observers. The proposed architecture deploys a force controller at the master side, responsible for transmitting the reflected slave forces to the master manipulator. The master states (position, velocity, and acceleration) are transmitted to the slave side for tracking of the master trajectory by the slave. The environmental force acting on the slave is transmitted back to the master side. In this scenario, 
the slave manipulator is operated to track the trajectory of the master manipulator, while at the master side, the control objective is to apply the reflected force and make the environment force follow the desired operator force. When these goals are accomplished, in the ideal case with no time delays, the master operator would experience the same interaction with the environment as the slave manipulator ${ }^{10}$.

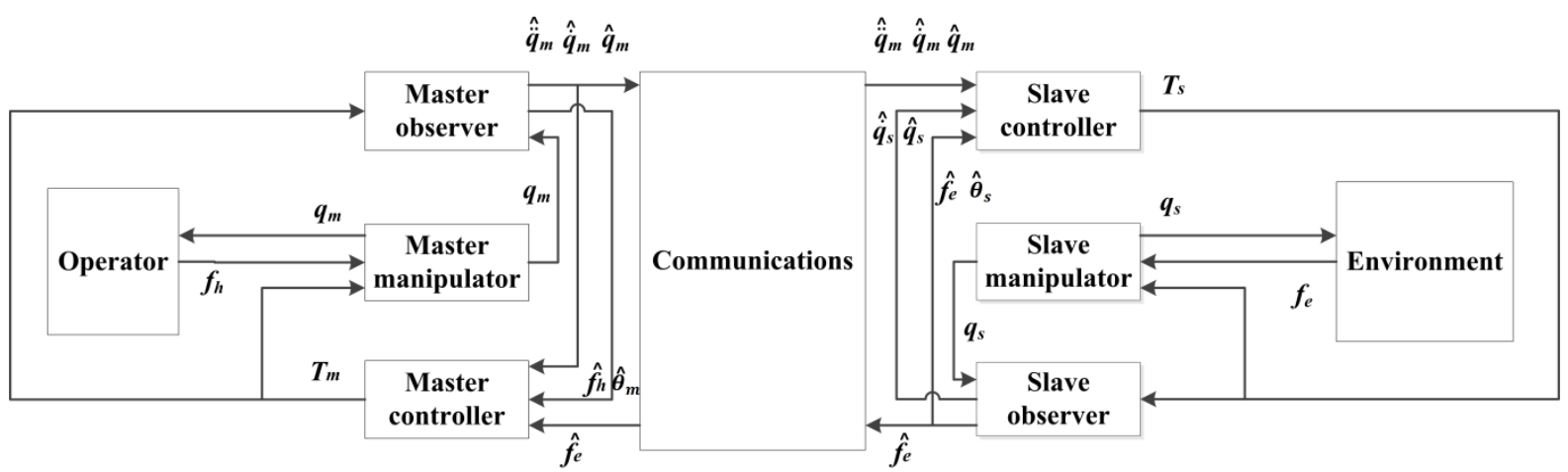

Fig. 1. Block diagram of the entire teleoperation system

\subsection{Proposed EAOB}

In this Section, the proposed Extended Active Observer for simultaneous system state, parameter and force estimation is presented. Then, the stability of the EAOB is analyzed.

\subsubsection{The EAOB}

An Active Observer (AOB) ${ }^{11,12}$ is a variation of a Kalman filter (KF), one of the first estimators to include disturbance in the optimization process. The AOB concept relies on adopting an extra relationship (auxiliary input) to estimate an equivalent disturbance referred to as the system input. The active state $p_{k}$ describing the equivalent disturbance is defined by

$$
p_{k}=\sum_{j=1}^{S}(-1)^{j+1} \frac{S !}{j !(S-j) !} p_{k-j}+{ }^{S-1} \xi_{p_{k^{\prime}}}
$$

where the Sth-order derivative of $p_{k}$ is randomly distributed. ${ }^{S-1} \xi_{p_{k}}$ is a Gaussian variable with zero mean.

Considering the continuous time of (5), when $S=1$, we have $p_{k}=p_{k-1}+{ }^{S-1} \xi_{p_{k}}$, then the continuous time of (5) can be written as

$$
\dot{p}=\frac{p_{k}-p_{k-1}}{T_{s}}=0+\frac{1}{T_{s}}{ }^{0} \xi_{p_{k}}
$$

likewise, we can deduce, 
when $S=2$,

when $S=3$,

$$
\ddot{p}=0+\frac{1}{T_{s}}{ }^{1} \xi_{p_{k}}
$$

$$
\dddot{p}=0+\frac{1}{T_{s}}{ }^{2} \xi_{p_{k^{\prime}}}
$$

Where $T_{s}$ is the sampling period.

As indicated in ref. [11, 12], $p$ can be used to estimate the disturbance in a dynamic system, as well as estimating unknown input functions. In this work, by treating the external forces acting on a manipulator as unknown inputs $\left(p=f_{h} / f_{e}\right.$ ), those forces can be recovered by the active observer. Meanwhile, considering that the robot dynamics is nonlinear with inertial parameter uncertainties, by defining the state vector $X_{*}(*=m / s)$ as

$$
X_{*}=\left[\begin{array}{c}
q_{*} \\
\dot{q}_{*} \\
f_{h / e} \\
\theta_{*}
\end{array}\right],
$$

the teleoperation system model in (3) can be extended as follows:

$$
\begin{aligned}
& \dot{X}_{*}=f_{*}\left(X_{*}, T_{*}\right)+G_{*} \xi_{X_{*}}=\left[\begin{array}{c}
\dot{q}_{*} \\
M_{*}^{-1}\left(-V_{*} \dot{q}_{*}-g_{*}+T_{*}-f_{h / e}\right) \\
0 \\
0
\end{array}\right]+G_{*}\left[\begin{array}{c}
\xi_{q_{*}} \\
\xi_{\dot{q}_{*}} \\
\xi_{f_{h / e}} \\
\xi_{\theta_{*}}
\end{array}\right], \\
& Y_{*}=H_{*} X_{*}+\eta_{X_{*}}=\left[\begin{array}{llll}
I & 0 & 0 & 0
\end{array}\right]\left[\begin{array}{c}
q_{*} \\
\dot{q}_{*} \\
f_{h / e} \\
\theta_{*}
\end{array}\right]+\eta_{X_{*}}=q_{*}+\eta_{X_{*}}
\end{aligned}
$$

where $Y_{*}$ is the output of the system, $G_{*}$ is a unit matrix, and the state observation matrix $H_{*}=\left[\begin{array}{llll}I & 0 & 0 & 0\end{array}\right]$, and $\xi_{q_{*}}, \xi_{\dot{q}_{*}}$ and $\eta_{X_{*}}$ represent the process noises and measurement noises, respectively, ${ }^{0} \xi_{f_{h / e}}$ and $\xi_{\theta_{*}}$ represent the rates at which the vectors of external forces and inertial robot parameters are estimated to vary.

Therefore, the extended active observer (EAOB) for inertial parameter estimation and external force estimation for a nonlinear robotic system (either the master or the slave manipulator), modeled in (7), is proposed as follows (Take $S=1$ for example)

$$
\dot{\hat{X}}_{*}=f_{*}\left(\hat{X}_{*}, T_{*}\right)+P_{*} H_{*}^{T} R_{*}^{-1}\left(Y_{*}-H_{*} \hat{X}_{*}\right)
$$


where

$$
\dot{P}_{*}=\frac{\partial f_{*}}{\partial \hat{X}_{*}} P_{*}+P_{*} \frac{\partial f_{*}^{T}}{\partial \hat{X}_{*}}+G_{*} Q_{*} G_{*}^{T}-P_{*} H_{*}^{T} R_{*}^{-1} H_{*} P_{*}
$$

and

$$
\begin{gathered}
R_{*}=\operatorname{cov}\left(\eta_{X_{*}}\right), \\
Q_{*}=\left[\begin{array}{cccc}
\operatorname{cov}\left(\xi_{q_{*}}\right) & 0 & 0 & 0 \\
0 & \operatorname{cov}\left(\xi_{\dot{q}_{*}}\right) & 0 & 0 \\
0 & 0 & \operatorname{cov}\left({ }^{0} \xi_{f_{h / e}}\right) & \operatorname{cov}\left(\xi_{\theta_{*}}\right)
\end{array}\right], \\
f_{*}\left(\hat{X}_{*}, T_{*}\right)=\left[\begin{array}{c}
\hat{\hat{q}}_{*} \\
\hat{\hat{\hat{q}}}_{*} \\
\hat{\hat{f}}_{h / e} \\
\hat{\hat{\theta}}_{*}
\end{array}\right]=\left[\begin{array}{c}
\hat{M}_{*}^{-1}\left(-\hat{V}_{*} \hat{\hat{q}}_{*}-\hat{\hat{q}}_{*}+T_{*}-\hat{f}_{h / e}\right) \\
0 \\
0
\end{array}\right],
\end{gathered}
$$

where $\operatorname{cov}\left(\xi_{q_{*}}\right), \operatorname{cov}\left(\xi_{\dot{q}_{*}}\right), o v\left({ }^{0} \xi_{f_{h / e}}\right), \operatorname{cov}\left(\xi_{\theta_{*}}\right)$, and $\operatorname{cov}\left(\eta_{X_{*}}\right)$ are, respectively, the covariance matrices of the input stochastic, zero mean, and Gaussian noises $\xi_{q_{*}}, \xi_{\dot{q}_{*}},{ }^{0} \xi_{f_{h / e^{e}}} \xi_{\theta_{*}}$, and the output stochastic, zero mean, and Gaussian noise $\eta_{X_{*}}$, and

$$
F_{*}(t)=\frac{\partial f_{*}}{\partial X_{*}}=\left[\begin{array}{ccrl}
0 & I & 0 & 0 \\
F_{* 21}(t) & F_{* 22}(t) & F_{* 23}(t) & F_{* 24}(t) \\
0 & 0 & 0 & 0 \\
0 & 0 & 0 & 0
\end{array}\right],
$$

where

$$
\begin{gathered}
F_{* 21}(t)=M_{*}^{-1}\left(\frac{\partial M_{*}}{\partial \hat{q}_{*}} \ddot{\hat{q}}_{*}+\frac{\partial V_{*} \dot{\hat{q}}_{*}}{\partial \hat{q}_{*}}+\frac{\partial \hat{g}_{*}}{\partial \hat{q}_{*}} \mp \frac{\partial J J_{*}^{T}\left(\hat{q}_{*}\right) \hat{f}_{h / e}}{\partial \hat{q}_{*}}\right), \\
F_{* 22}(t)=-M_{*}^{-1} \frac{\partial V_{*} \dot{\hat{q}}_{*}}{\partial \dot{\hat{q}}_{*}}, \\
F_{* 23}(t)=\mp M_{*}^{-1} J_{*}^{T}\left(\hat{q}_{*}\right), \\
F_{* 24}(t)=-\widehat{M}_{*}^{-1}\left(\frac{\partial \widehat{M}_{*}}{\partial \hat{\theta}_{*}} \ddot{\hat{q}}_{*}+\frac{\partial \widehat{V}_{*} \dot{\hat{q}}_{*}}{\partial \hat{\theta}_{*}}+\frac{\partial \hat{g}_{*}}{\partial \hat{\theta}_{*}}\right) .
\end{gathered}
$$

\subsubsection{Stability analysis of the EAOB}


Since AOB extends the Kalman filter (KF) by adding another estimated variable along with the system states to estimate the disturbance, it can be viewed as an Extended Kalman Filter (EKF). Similarly, the proposed EAOB extends the AOB by further employing another variable to estimate robot inertial parameters, so the EAOB can be also seen as another EKF.

In ref. [7,13], an EKF is designed for nonlinear robot systems. Robot inertial parameters are added into the estimated variables of the EKF, and a solid stability analysis for the EKF is provided. The EAOB proposed in this work can be regarded as an extension of the EKF, and the stability analysis of EAOB for nonlinear robot manipulators can be carried out based on Theorem 1, which is an extension of the results produced in ref. [13].

Theorem 1 The filter described by equations (8)-(9) for the system modeled by (7) is locally stable, provided that

1. $\alpha_{1} I \leq Q_{*}(t) \leq \alpha_{2} I$,

2. $\alpha_{3} I \leq R_{*}(t) \leq \alpha_{4} I$,

3. the following is true:

$$
\alpha_{5} I \leq \int_{t}^{t+\sigma}\left[F_{* 23}(\tau) \quad F_{* 24}(\tau)\right]^{T}\left[F_{* 23}(\tau) \quad F_{* 24}(\tau)\right] d \tau \leq \alpha_{6} I
$$

Where $F_{* 23}(\tau)$ and $F_{* 24}(\tau)$ are evaluated along $\hat{X}_{*}$ and $\dot{F}_{* 23}(\tau)$ and $\dot{F}_{* 24}(\tau)$ are bounded, with

$$
\begin{gathered}
F_{* 23}(\tau)=\left[\mp \widehat{M}_{*}^{-1} \hat{J}_{*}^{T}\right]_{\text {along } \hat{X}_{*}} \\
F_{* 24}(\tau)=\left[-\widehat{M}_{*}^{-1}\left(\frac{\partial \widehat{M}_{*}}{\partial \hat{\theta}_{*}} \ddot{\hat{q}}_{*}+\frac{\partial \hat{V}_{*} \dot{\hat{q}}_{*}}{\partial \hat{\theta}_{*}}+\frac{\partial \hat{g}_{*}}{\partial \hat{\theta}_{*}}\right)\right]_{\text {along } \hat{X}_{*}}
\end{gathered}
$$

for some positive constants $\alpha_{1}, \alpha_{2}, \alpha_{3}, \alpha_{4}, \alpha_{5}, \alpha_{6}, \sigma$ and all $t>t_{0}$.

Proof outline: The stability analysis of the EAOB is established based on Theorem 2:

Theorem 2 Assume that the model of a linear system is

1. uniformly completely observable,

2. uniformly completely controllable, 
3. $\alpha_{1} \leq\|Q(t)\| \leq \alpha_{2}$,

4. $\alpha_{3} \leq\|R(t)\| \leq \alpha_{4}$,

5. $\|F(t)\| \leq \alpha_{5},\|G(t)\| \leq \alpha_{6},\|H(t)\| \leq \alpha_{7}$,

then the following is true: the optimal filter $(\tilde{X}=X-\hat{X})$

$$
\begin{gathered}
\dot{\hat{X}}=F(t) \hat{X}+P(t) H^{T} R^{-1}(t) H \tilde{X}, \\
\frac{d \tilde{X}}{d t}=\left[F(t)-P(t) H^{T}(t) R^{-1}(t) H(t)\right] \tilde{X},
\end{gathered}
$$

is uniformly asymptotically stable.

Proof can be found in ref. [14].

According to Theorem 2, the nonlinear robot dynamics in (7) should be first linearized. By using a first order Taylor series expansion and ignoring higher order terms, the nonlinear system can be linearized as follows:

$$
\begin{gathered}
\dot{X}_{*}=F_{*}(t) X_{*}+G_{*} \xi_{X_{*}^{\prime}} \\
Y_{*}=H_{*} X_{*}+\eta_{X_{*}^{\prime}}
\end{gathered}
$$

where $F_{*}(t)$ is described in (9). Then, the stability of the EAOB can be achieved through the following four steps. 1) shows that under the conditions 2 and 3 of Theorem 1 the system described in (10) is totally observable, 2) shows that under the condition 1 of Theorem 1 the system described in (10) is totally controllable. Then, 3) states the results of Theorem 2, and 4) shows local stability even if the linearization is not exact.

Proof: See ref. [13]. The different part between stability analysis in this paper and that in ref. [13] is to replace " $F_{* 23}(\tau), x_{3}=\theta_{*}, G_{*}=\left[\begin{array}{cc}0 & 0 \\ \widehat{M}_{*}^{-1} & 0 \\ 0 & I\end{array}\right]$ " with “ $\left[F_{* 23}(\tau) \quad F_{* 24}(\tau)\right], x_{3}=\left[\begin{array}{ll}f_{h / e} & \theta_{*}\end{array}\right], G_{*}=I$ " in the paper, which does not affect stability analysis.

\subsection{The teleoperation control laws}

Using inverse dynamics ${ }^{15}$, the driving torque for the master manipulator based on estimated signals is proposed as 


$$
T_{m}=M_{m}\left(\hat{q}_{m}, \widehat{\theta}_{m}\right) a_{m}+V_{m}\left(\hat{q}_{m}, \dot{\hat{q}}_{m}, \widehat{\theta}_{m}\right) \dot{\hat{q}}_{m}+g_{m}\left(\hat{q}_{m}, \widehat{\theta}_{m}\right)-J_{m}^{T}\left(\hat{q}_{m}\right) \hat{f}_{h}
$$

where $a_{m}$ is the control law, and $\hat{f}_{h}$ is estimated operator force.

Similarly, the driving torque for the slave manipulator is designed as

$$
T_{s}=M_{s}\left(\hat{q}_{s}, \widehat{\theta}_{s}\right) a_{s}+V_{s}\left(\hat{q}_{s}, \dot{\hat{q}}_{s}, \widehat{\theta}_{s}\right) \dot{\hat{q}}_{s}+g_{s}\left(\hat{q}_{s}, \widehat{\theta}_{s}\right)+J_{s}^{T}\left(\hat{q}_{s}\right) \hat{f}_{e}
$$

where $a_{s}$ is the control law, and $\hat{f}_{e}$ is estimated environment force.

There are different options available for the control law $a_{*}(*=m / s)$ at the master and slave sides.

\subsubsection{Force controller at the master side}

In this work, the master robot manipulator of the teleoperation system is controlled to track a given operator' force $f_{h}$, assigned in the joint space. Thus, in order to eliminate the force error at steady state, an integral action on the force error is introduced. Furthermore, an inner loop on the end-effector position is added. Hence, the new control input in (11) is chosen as

$$
a_{m}=-K_{m d} \dot{\hat{q}}_{m}+K_{m p}\left(q_{f}-\hat{q}_{m}\right)
$$

where

$$
q_{f}=K_{m c} J_{m}^{T}\left(\hat{q}_{m}\right)\left(\hat{f}_{h}-\hat{f}_{e}\right)+K_{m i c} \int_{0}^{t} J_{m}^{T}\left(\hat{q}_{m}\right)\left(\hat{f}_{h}-\hat{f}_{e}\right) d \tau
$$

where $K_{m d}, K_{m p}, K_{m c}$, and $K_{m i c}$ are force controller gain matrices.

\subsubsection{Position controller at the slave side}

The slave robot manipulator of the teleoperation system is controlled to track the trajectory of the master robot manipulator $q_{m}$, and a position control scheme of PD type is chosen as the control method. Thus, the new control input in (12) is chosen as

$$
a_{s}=\ddot{\hat{q}}_{m}+K_{s d}\left(\dot{\hat{q}}_{m}-\dot{\hat{q}}_{s}\right)+K_{s p}\left(\hat{q}_{m}-\hat{q}_{s}\right)
$$

where $\ddot{\hat{q}}_{m}$ is estimated acceleration of the master manipulator, $\dot{\hat{q}}_{*}$ and $\hat{q}_{*}(*=m / s)$ are estimated velocity and position signals of the master/slave manipulator, and $K_{s d}$ and $K_{s p}$ are position controller gain matrices. 


\subsection{Stability analysis of the teleoperation system}

A teleoperation system consists of five interconnected parts: operator, master device, communication channel, slave robot, and environment. Since the operator and environment are commonly assumed to be passive, they are stable. Furthermore, the communication between the master and salve sides is assumed to be ideal in this work, so it is also stable. Therefore, we will show that under the proposed control laws and EAOB the master and slave sides are stable using Theorem 3 in order to ensure the stability of the entire teleoperation system.

Theorem 3 In the teleoperation system described by equations (7) with the control laws (11)-(14) and the EAOB (8)-(9), the velocities $\dot{q}_{m}, \dot{q}_{s}$ and position error $q_{m}-q_{s}$ are bounded $\left(\dot{q}_{m}, \dot{q}_{s}, q_{m}-q_{s} \in L_{2} \cap L_{\infty}\right)$, provided that

1. $M_{m}\left(\hat{q}_{m}, \hat{\theta}_{m}\right), M_{s}\left(\hat{q}_{s}, \hat{\theta}_{s}\right), J_{m}\left(\hat{q}_{m}\right)$ and $J_{s}\left(\hat{q}_{s}\right)$ are positive definite matrices,

2. $f_{h}, f_{e}$ are bounded and continuous,

3. $K_{s d}, K_{s p}, K_{m d}, K_{m p}, K_{m c}$, and $K_{m i c}$ are bounded and positive definite matrices,

4. $\alpha_{1} I \leq Q_{m}(t) \leq \alpha_{2} I, \beta_{1} I \leq Q_{s}(t) \leq \beta_{2} I$,

5. $\alpha_{3} I \leq R_{m}(t) \leq \alpha_{4} I, \beta_{3} I \leq R_{S}(t) \leq \beta_{4} I$,

6. the following is true:

$$
\alpha_{5} I \leq \int_{t}^{t+\sigma}\left[F_{m 23}(\tau) \quad F_{m 24}(\tau)\right]^{T}\left[F_{m 23}(\tau) \quad F_{m 24}(\tau)\right] d \tau \leq \alpha_{6} I
$$

where $F_{m 23}(\tau)$ and $F_{m 24}(\tau)$ are evaluated along $\hat{X}_{m}$ and $\dot{F}_{m 23}(\tau)$ and $\dot{F}_{m 24}(\tau)$ are bounded, with

$$
\begin{gathered}
F_{m 23}(\tau)=\left[-\widehat{M}_{m}^{-1} \hat{J}_{m}^{T}\right]_{\text {along } \hat{X}_{m}{ }^{\prime}} \\
F_{m 24}(\tau)=\left[-\widehat{M}_{m}^{-1}\left(\frac{\partial \widehat{M}_{m}}{\partial \hat{\theta}_{m}} \ddot{\hat{q}}_{m}+\frac{\partial \widehat{V}_{m} \dot{\hat{q}}_{m}}{\partial \hat{\theta}_{m}}+\frac{\partial \hat{g}_{m}}{\partial \hat{\theta}_{m}}\right)\right]_{\text {along } \hat{X}_{m}},
\end{gathered}
$$

for some positive constants $\alpha_{1}, \alpha_{2}, \alpha_{3}, \alpha_{4}, \alpha_{5}, \alpha_{6}, \sigma$ and all $t>t_{0}$,

7. the following is true: 


$$
\beta_{5} I \leq \int_{t}^{t+\sigma}\left[F_{s 23}(\tau) \quad F_{s 24}(\tau)\right]^{T}\left[F_{s 23}(\tau) \quad F_{s 24}(\tau)\right] d \tau \leq \beta_{6} I,
$$

where $F_{s 23}(\tau)$ and $F_{s 24}(\tau)$ are evaluated along $\hat{X}_{s}$ and $\dot{F}_{s 23}(\tau)$ and $\dot{F}_{s 24}(\tau)$ are bounded, with

$$
\begin{gathered}
F_{s 23}(\tau)=\left[\widehat{M}_{s}^{-1} \hat{J}_{s}^{T}\right]_{\text {along } \hat{X}_{s}{ }^{\prime}} \\
F_{s 24}(\tau)=\left[-\widehat{M}_{s}^{-1}\left(\frac{\partial \widehat{M}_{s}}{\partial \hat{\theta}_{s}} \ddot{\hat{q}}_{s}+\frac{\partial \widehat{V}_{s} \dot{\hat{q}}_{s}}{\partial \hat{\theta}_{s}}+\frac{\partial \hat{g}_{s}}{\partial \hat{\theta}_{s}}\right)\right]_{\text {along } \hat{X}_{s}},
\end{gathered}
$$

for some positive constants $\beta_{1}, \beta_{2}, \beta_{3}, \beta_{4}, \beta_{5}, \beta_{6}, \sigma$ and all $t>t_{0}$.

Proof: Define

$$
e=q_{m}-q_{s}, \tilde{\tilde{q}}_{*}=\ddot{q}_{*}-\hat{\tilde{q}}_{*}, \tilde{\dot{q}}_{*}=\dot{q}_{*}-\hat{\dot{q}}_{*}, \tilde{q}_{*}=q_{*}-\hat{q}_{*}, \tilde{\theta}_{*}=\theta_{*}-\hat{\theta}_{*}, \tilde{\tilde{f}}_{h / e}=\dot{f}_{h / e}-\hat{\hat{f}}_{h / e},
$$

where $*=m / s$. If conditions $4,5,6,7$ of the Theorem are satisfied, according to Theorem 1 , $\tilde{q}_{m}, \tilde{q}_{m}, \tilde{\theta}_{m}, \tilde{f}_{h}, \tilde{q}_{s}, \tilde{q}_{s}, \tilde{\theta}_{s}, \tilde{f}_{e} \in L_{2} \cap L_{\infty}$.

1) We first consider the master side, and show that $\ddot{q}_{m}, \dot{q}_{m}, q_{m} \in L_{2} \cap L_{\infty}$.

The master system is given by

$$
T_{m}=M_{m}\left(q_{m}, \theta_{m}\right) \ddot{q}_{m}+V_{m}\left(q_{m}, \dot{q}_{m}, \theta_{m}\right) \dot{q}_{m}+g_{m}\left(q_{m}, \theta_{m}\right)-J_{m}^{T}\left(q_{m}\right) f_{h}=Y_{1}\left(q_{m}, \dot{q}_{m}, \ddot{q}_{m}\right) x_{k},
$$

where $x_{k}=\left[\theta_{m}, f_{h}\right]^{T}$, and the master controller is given by

$$
\begin{aligned}
T_{m}=M_{m}\left(\hat{q}_{m}, \hat{\theta}_{m}\right) & \left\{-K_{m d} \dot{\hat{q}}_{m}-K_{m p} \hat{q}_{m}+K_{m p}\left(K_{m c} J_{m}^{T}\left(\hat{q}_{m}\right)\left(\hat{f}_{h}-\hat{f}_{e}\right)+K_{m i c} \int_{0}^{t} J_{m}^{T}\left(\hat{q}_{m}\right)\left(\hat{f}_{h}-\hat{f}_{e}\right) d \tau\right)\right\} \\
& +V_{m}\left(\hat{q}_{m}, \dot{\hat{q}}_{m}, \hat{\theta}_{m}\right) \dot{\hat{q}}_{m}+g_{m}\left(\hat{q}_{m}, \hat{\theta}_{m}\right)+J_{m}^{T}\left(\hat{q}_{m}\right) \hat{f}_{h} \\
& =Y_{1}\left(\hat{q}_{m}, \dot{\hat{q}}_{m}, \ddot{q}_{m}\right) \hat{x}_{k} \\
& +M_{m}\left(\hat{q}_{m}, \hat{\theta}_{m}\right)\left\{-\ddot{q}_{m}-K_{m d} \dot{\hat{q}}_{m}-K_{m p} \hat{q}_{m}+K_{m p}\left(K_{m c} J_{m}^{T}\left(\hat{q}_{m}\right)\left(\hat{f}_{h}-\hat{f}_{e}\right)\right.\right. \\
& \left.\left.+K_{m i c} \int_{0}^{t} J_{m}^{T}\left(\hat{q}_{m}\right)\left(\hat{f}_{h}-\hat{f}_{e}\right) d \tau\right)\right\} .
\end{aligned}
$$

Then, one can get 


$$
\begin{gathered}
M_{m}\left(\hat{q}_{m}, \hat{\theta}_{m}\right)\left\{-\ddot{q}_{m}-K_{m d} \dot{\hat{q}}_{m}-K_{m p} \hat{q}_{m}+K_{m p}\left(K_{m c} J_{m}^{T}\left(\hat{q}_{m}\right)\left(\hat{f}_{h}-\hat{f}_{e}\right)+K_{m i c} \int_{0}^{t} J_{m}^{T}\left(\hat{q}_{m}\right)\left(\hat{f}_{h}-\hat{f}_{e}\right) d \tau\right)\right\} \\
=Y_{1}\left(q_{m}, \dot{q}_{m}, \ddot{q}_{m}\right) x_{k}-Y_{1}\left(\hat{q}_{m}, \dot{\hat{q}}_{m}, \ddot{q}_{m}\right) \hat{x}_{k},
\end{gathered}
$$

which gives us

$$
\begin{aligned}
K_{m p}\left(K_{m c} J_{m}^{T}\left(\hat{q}_{m}\right)\right. & \left.\left(\hat{f}_{h}-\hat{f}_{e}\right)+K_{m i c} \int_{0}^{t} J_{m}^{T}\left(\hat{q}_{m}\right)\left(\hat{f}_{h}-\hat{f}_{e}\right) d \tau\right) \\
= & \widehat{M}_{m}^{-1}\left(\hat{q}_{m}, \hat{\theta}_{m}\right)\left\{Y_{1}\left(q_{m}, \dot{q}_{m}, \ddot{q}_{m}\right) \tilde{x}_{k}+\left(Y_{1}\left(q_{m}, \dot{q}_{m}, \ddot{q}_{m}\right)-Y_{1}\left(\hat{q}_{m}, \dot{\hat{q}}_{m}, \ddot{q}_{m}\right)\right) \hat{x}_{k}\right\} \\
& +\widehat{M}_{m}^{-1}\left(\hat{q}_{m}, \hat{\theta}_{m}\right)\left\{\ddot{q}_{m}+K_{m d} \dot{q}_{m}+K_{m p} q_{m}-K_{m d} \dot{\tilde{q}}_{m}-K_{m p} \tilde{q}_{m}\right\} .
\end{aligned}
$$

Now, if $\tilde{q}_{m}, \tilde{\dot{q}}_{m}, \tilde{\theta}_{m}, \tilde{f}_{h} \in L_{2} \cap L_{\infty}$ and $M_{m}\left(\hat{q}_{m}, \hat{\theta}_{m}\right), J_{m}\left(\hat{q}_{m}\right), K_{m d}, K_{m p}, K_{m c}$, and $K_{m i c}$ are positive definite, we have $\widehat{M}_{m}^{-1}\left(\hat{q}_{m}, \hat{\theta}_{m}\right)\left\{-K_{m d} \dot{\tilde{q}}_{m}-K_{m p} \tilde{q}_{m}\right\} \in L_{2} \cap L_{\infty}, Y_{1}\left(q_{m}, \dot{q}_{m}, \ddot{q}_{m}\right) \in L_{\infty}, Y_{1}\left(q_{m}, \dot{q}_{m}, \ddot{q}_{m}\right) \tilde{x}_{k} \in L_{2} \cap L_{\infty}$, and because PI control is exerted on the force error and $f_{h}, f_{e}$ are bounded and continuous, $K_{m p}\left(K_{m c}\right)_{m}^{T}\left(\hat{q}_{m}\right)\left(f_{h}-\right.$ $\left.\left.f_{e}-\tilde{f}_{h}+\tilde{f}_{e}\right)+K_{m i c} \int_{0}^{t} J_{m}^{T}\left(\hat{q}_{m}\right)\left(f_{h}-f_{e}-\tilde{f}_{h}+\tilde{f}_{e}\right) d \tau\right) \in L_{2} \cap L_{\infty}$. Furthermore, $Y_{1}\left(q_{m}, \dot{q}_{m}, \ddot{q}_{m}\right)$ is continuous in $q_{m}, \dot{q}_{m}$, has continuous and bounded partial derivatives with respect to $q_{m}, \dot{q}_{m}$, and satisfies a local Lipschitz condition:

$$
\left\|Y_{1}\left(q_{m}, \dot{q}_{m}, \ddot{q}_{m}\right)-Y_{1}\left(\hat{q}_{m}, \dot{\hat{q}}_{m}, \ddot{q}_{m}\right)\right\| \leq k_{1}\left\|\left[\begin{array}{c}
q_{m}-\hat{q}_{m} \\
\dot{q}_{m}-\hat{\dot{q}}_{m}
\end{array}\right]\right\|
$$

for some $0<k_{1}<\infty$. Hence, $Y_{1}\left(q_{m}, \dot{q}_{m}, \ddot{q}_{m}\right)-Y_{1}\left(\hat{q}_{m}, \dot{\hat{q}}_{m}, \ddot{q}_{m}\right) \in L_{2} \cap L_{\infty}, \hat{x}_{k} \in L_{\infty}$, and $\left[Y_{1}\left(q_{m}, \dot{q}_{m}, \ddot{q}_{m}\right)-\right.$ $\left.Y_{1}\left(\hat{q}_{m}, \dot{\hat{q}}_{m}, \ddot{q}_{m}\right)\right] \hat{x}_{k} \in L_{2} \cap L_{\infty}$. Therefore,

$$
\ddot{q}_{m}+K_{m d} \dot{q}_{m}+K_{m p} q_{m} \in L_{2} \cap L_{\infty}
$$

which implies that $\ddot{q}_{m}, \dot{q}_{m}, q_{m} \in L_{2} \cap L_{\infty}$.

2) We now consider the slave side, and show that $\dot{q}_{s}, e=q_{m}-q_{s} \in L_{2} \cap L_{\infty}$.

The slave system is given by

$$
T_{s}=M_{s}\left(q_{s}, \theta_{s}\right) \ddot{q}_{s}+V_{s}\left(q_{s}, \dot{q}_{s}, \theta_{s}\right) \dot{q}_{s}+g_{s}\left(q_{s}, \theta_{s}\right)+J_{s}^{T}\left(q_{s}\right) f_{e}=Y_{2}\left(q_{s}, \dot{q}_{s}, \ddot{q}_{s}\right) x_{l}
$$

where $x_{l}=\left[\theta_{s}, f_{e}\right]^{T}$, and the slave controller is given by 


$$
\begin{array}{r}
T_{s}=M_{s}\left(\hat{q}_{s}, \hat{\theta}_{s}\right)\left\{\ddot{\hat{q}}_{m}+K_{s d}\left(\dot{\hat{q}}_{m}-\dot{\hat{q}}_{s}\right)+K_{s p}\left(\hat{q}_{m}-\hat{q}_{s}\right)\right\}+V_{s}\left(\hat{q}_{s}, \dot{\hat{q}}_{s}, \hat{\theta}_{s}\right) \dot{\hat{q}}_{s}+g_{s}\left(\hat{q}_{s}, \hat{\theta}_{s}\right)+J_{s}^{T}\left(\hat{q}_{s}\right) \hat{f}_{e} \\
=Y_{2}\left(\hat{q}_{s}, \dot{\hat{q}}_{s}, \ddot{q}_{s}\right) \hat{x}_{l}+M_{s}\left(\hat{q}_{s}, \hat{\theta}_{s}\right)\left\{\left(\ddot{\hat{q}}_{m}-\ddot{q}_{s}\right)+K_{s d}\left(\dot{\hat{q}}_{m}-\dot{\hat{q}}_{s}\right)+K_{s p}\left(\hat{q}_{m}-\hat{q}_{s}\right)\right\} .
\end{array}
$$

Then, on can get

$$
M_{s}\left(\hat{q}_{s}, \hat{\theta}_{s}\right)\left\{\left(\ddot{e}-\tilde{q}_{m}\right)+K_{s d}\left(\dot{e}-\dot{\tilde{q}}_{m}+\dot{\tilde{q}}_{s}\right)+K_{s p}\left(e-\tilde{q}_{m}+\tilde{q}_{s}\right)\right\}=Y_{2}\left(q_{s}, \dot{q}_{s}, \ddot{q}_{s}\right) x_{l}-Y_{2}\left(\hat{q}_{s}, \dot{\hat{q}}_{s}, \ddot{q}_{s}\right) \hat{x}_{l},
$$

which gives us

$$
\begin{aligned}
\ddot{e}+K_{s d} \dot{e}+K_{s p} e & =\widehat{M}_{s}^{-1}\left(\hat{q}_{s}, \hat{\theta}_{s}\right)\left\{Y_{2}\left(q_{s}, \dot{q}_{s}, \ddot{q}_{s}\right) x_{l}-Y_{2}\left(\hat{q}_{s}, \dot{\hat{q}}_{s}, \ddot{q}_{s}\right) \hat{x}_{l}\right\}+\tilde{q}_{m}+K_{s d}\left(\dot{\tilde{q}}_{m}-\dot{\tilde{q}}_{s}\right)+K_{s p}\left(\tilde{q}_{m}-\tilde{q}_{s}\right) \\
& =\widehat{M}_{s}^{-1}\left(\hat{q}_{s}, \hat{\theta}_{s}\right)\left\{Y_{2}\left(q_{s}, \dot{q}_{s}, \ddot{q}_{s}\right) \tilde{x}_{l}+\left(Y_{2}\left(q_{s}, \dot{q}_{s}, \ddot{q}_{s}\right)-Y_{2}\left(\hat{q}_{s}, \dot{\hat{q}}_{s}, \ddot{q}_{s}\right)\right) \hat{x}_{l}\right\}+\tilde{q}_{m}+K_{s d}\left(\dot{\tilde{q}}_{m}-\dot{\tilde{q}}_{s}\right) \\
& +K_{s p}\left(\tilde{q}_{m}-\tilde{q}_{s}\right) .
\end{aligned}
$$

Now, if $\tilde{q}_{m}, \tilde{q}_{m}, \tilde{q}_{m}, \tilde{q}_{s}, \tilde{\dot{q}}_{s}, \tilde{\theta}_{s}, \tilde{f}_{e} \in L_{2} \cap L_{\infty}$ and $M_{s}\left(\hat{q}_{s}, \hat{\theta}_{s}\right), K_{s d}$ and $K_{s p}$ are positive definite, we have $K_{s d}\left(\dot{\tilde{q}}_{m}-\right.$ $\left.\dot{\tilde{q}}_{s}\right) \in L_{2} \cap L_{\infty}, \quad K_{s p}\left(\tilde{q}_{m}-\tilde{q}_{s}\right) \in L_{2} \cap L_{\infty}, Y_{2}\left(q_{s}, \dot{q}_{s}, \ddot{q}_{s}\right) \in L_{\infty}, Y_{2}\left(q_{s}, \dot{q}_{s}, \ddot{q}_{s}\right) \tilde{x}_{l} \in L_{2} \cap L_{\infty}$, and $Y_{2}\left(q_{s}, \dot{q}_{s}, \ddot{q}_{s}\right)$ is continuous in $q_{s}, \dot{q}_{s}$, has continuous and bounded partial derivatives with respect to $q_{s}, \dot{q}_{s}$, and satisfies a local Lipschitz condition:

$$
\left\|Y_{2}\left(q_{s}, \dot{q}_{s}, \ddot{q}_{s}\right)-Y_{2}\left(\hat{q}_{s}, \dot{\hat{q}}_{s}, \ddot{q}_{s}\right)\right\| \leq k_{2}\left\|\left[\begin{array}{c}
q_{s}-\hat{q}_{s} \\
\dot{q}_{s}-\hat{\hat{q}}_{s}
\end{array}\right]\right\|
$$

for some $0<k_{2}<\infty$. Hence, $\tilde{q}_{s}, \tilde{q}_{s}, \tilde{\theta}_{s}, \tilde{f}_{e} \in L_{2} \cap L_{\infty}$ imply that $Y_{2}\left(q_{s}, \dot{q}_{s}, \ddot{q}_{s}\right)-Y_{2}\left(\hat{q}_{s}, \dot{\hat{q}}_{s}, \ddot{q}_{s}\right) \in L_{2} \cap L_{\infty}, \hat{x}_{l} \in$ $L_{\infty}$, and $\left[Y_{2}\left(q_{s}, \dot{q}_{s}, \ddot{q}_{s}\right)-Y_{2}\left(\hat{q}_{s}, \dot{\hat{q}}_{s}, \ddot{q}_{s}\right)\right] \hat{x}_{l} \in L_{2} \cap L_{\infty}$. Therefore,

$$
\ddot{e}+K_{s d} \dot{e}+K_{s p} e \in L_{2} \cap L_{\infty},
$$

which implies that $\dot{e}, e \in L_{2} \cap L_{\infty}$. Since $\dot{q}_{m} \in L_{2} \cap L_{\infty}, \dot{q}_{s}=\dot{q}_{m}-\dot{e} \in L_{2} \cap L_{\infty}$.

\section{Simulation study}

In this Section, computer simulations will be carried out to illustrate the effectiveness of the proposed control scheme. The performance of EAOB based control scheme is also compared against a typical Nisocia observer based scheme. Both the master and slave robots are considered to be planar two-link manipulators.

\subsection{Dynamics of master and slave robot manipulators}


In this work, the master and slave robot manipulators for the teleoperation system are both chosen as a typical 2 DOF nonlinear robotic manipulator. The dynamic model of a 2 DOF nonlinear teleoperation system in the joint space is defined as

$$
\begin{gathered}
M_{m}\left(q_{m}, \theta_{m}\right) \ddot{q}_{m}+V_{m}\left(q_{m}, \dot{q}_{m}, \theta_{m}\right) \dot{q}_{m}+g_{m}\left(q_{m}, \theta_{m}\right)=T_{m}+J_{m}^{T}\left(q_{m}\right) f_{h^{\prime}} \\
M_{s}\left(q_{s}, \theta_{s}\right) \ddot{q}_{s}+V_{s}\left(q_{s}, \dot{q}_{s}, \theta_{s}\right) \dot{q}_{s}+g_{s}\left(q_{s}, \theta_{s}\right)=T_{s}-J_{s}^{T}\left(q_{s}\right) f_{e}
\end{gathered}
$$

where

$$
\begin{gathered}
\theta_{*}=\left[\begin{array}{l}
\theta_{* 1} \\
\theta_{* 2}
\end{array}\right]=\left[\begin{array}{l}
m_{* 1} l_{* 1}^{2} \\
m_{* 2} l_{* 2}^{2}
\end{array}\right], \\
M_{*}\left(q_{*}, \theta_{*}\right)=\left[\begin{array}{cc}
\theta_{* 1}+2 \theta_{* 2}+2 \theta_{* 2} \cos \left(q_{* 2}\right) & \theta_{* 2}+\theta_{* 2} \cos \left(q_{* 2}\right) \\
\theta_{* 2}+\theta_{* 2} \cos \left(q_{* 2}\right) & \theta_{* 2}
\end{array}\right], \\
V_{*}\left(q_{*}, \dot{q}_{*}, \theta_{*}\right)=\left[\begin{array}{cc}
-2 \theta_{* 2} \dot{q}_{* 2} \sin \left(q_{* 2}\right) & -\theta_{* 2} \dot{q}_{* 2} \sin \left(q_{* 2}\right) \\
\theta_{* 2} \dot{q}_{* 1} \sin \left(q_{* 2}\right) & 0
\end{array}\right], \\
J_{*}\left(q_{*}\right)=\left[\begin{array}{cc}
-l_{* 1} \sin \left(q_{* 1}\right)-l_{* 2} \sin \left(q_{* 1}+q_{* 2}\right) & -l_{* 2} \sin \left(q_{* 1}+q_{* 2}\right) \\
l_{* 1} \cos \left(q_{* 1}\right)+l_{* 2} \cos \left(q_{* 1}+q_{* 2}\right) & l_{* 2} \cos \left(q_{* 1}+q_{* 2}\right)
\end{array}\right],
\end{gathered}
$$

$*=m / s, l_{* 1}=l_{* 2}$ are the lengths of the first and the second links, $m_{* 1}$ and $m_{* 2}$ are the masses of the first and the second links, and we assume the robots operate in a horizontal plane, and as such $g_{*}\left(q_{*}, \theta_{*}\right)$ is zero. Meanwhile, the forward kinematics connecting the end effector coordinates with joint angles can be expressed as

$$
Z_{*}=\left[\begin{array}{l}
Z_{* 1} \\
Z_{* 2}
\end{array}\right]=\left[\begin{array}{l}
l_{* 1} \cos \left(q_{* 1}\right)+l_{* 2} \cos \left(q_{* 1}+q_{* 2}\right) \\
l_{* 1} \sin \left(q_{* 1}\right)+l_{* 2} \sin \left(q_{* 1}+q_{* 2}\right)
\end{array}\right]
$$

where $\left(Z_{* 1}, Z_{* 2}\right)$ is the position of the end effector in robot task space. We define system state vector $X$ as

$$
X_{*}=\left[\begin{array}{c}
q_{* 1} \\
q_{* 2} \\
\dot{q}_{* 1} \\
\dot{q}_{* 2} \\
f_{h / e 1} \\
f_{h / e 2} \\
\theta_{* 1} \\
\theta_{* 2}
\end{array}\right],
$$

Thus, according to EAOB $(S=1)$, the master or slave side of the teleoperation system can be written as 


$$
\begin{gathered}
\dot{X}_{*}=\left[\begin{array}{c}
\dot{q}_{* 1} \\
\dot{q}_{* 2} \\
\ddot{q}_{* 1} \\
\ddot{q}_{* 2} \\
\dot{f}_{h / e 1} \\
\dot{f}_{h / e 2} \\
\dot{\theta}_{* 1} \\
\dot{\theta}_{* 2}
\end{array}\right]=f_{*}\left(X_{*}, T_{*}\right)+G_{*} \xi_{*}=\left[\begin{array}{c}
\dot{q}_{* 1} \\
\dot{q}_{* 2} \\
M_{*}^{-1}\left(-V_{*}\left[\begin{array}{c}
\dot{q}_{* 1} \\
\dot{q}_{* 2}
\end{array}\right]+\left[\begin{array}{c}
T_{* 1} \\
T_{* 2}
\end{array}\right]-J_{*}^{T}\left[\begin{array}{c}
f_{h / e 1} \\
0 \\
f_{h / e 2}
\end{array}\right]\right) \\
0 \\
0 \\
0
\end{array}\right]+G_{*}\left[\begin{array}{c}
\xi_{q_{* 1}} \\
\xi_{q_{* 2}} \\
\xi_{\dot{q}_{* 1}} \\
\xi_{\dot{q}_{* 2}} \\
\xi_{f_{h / e 1}} \\
\xi_{f / e 2} \\
\xi_{\theta_{* 1}} \\
\xi_{\theta_{* 2}}
\end{array}\right], \\
Y_{*}=H_{*} X_{*}+\eta_{*}=\left[\begin{array}{c}
q_{* 1} \\
q_{* 2}
\end{array}\right]+\left[\begin{array}{c}
\eta_{* 1} \\
\eta_{* 2}
\end{array}\right] .
\end{gathered}
$$

where $\xi_{q_{* 1}}, \xi_{q_{* 2}}, \xi_{\dot{q}_{* 1}}, \xi_{\dot{q}_{* 2}}$ and $\eta_{* 1}, \eta_{* 2}$ are Gaussian noise signals representing the process noises and the measurement noises, respectively, $\xi_{f_{h / e 1}}, \xi_{f_{h / e 2}}$ and $\xi_{\theta_{* 1}}, \xi_{\theta_{* 2}}$ represent the rates at which the vectors of external forces and inertial robot parameters are estimated to vary, and

$$
\begin{gathered}
G_{*}=\operatorname{diag}\{1,1,1,1,1,1,1,1\}, \\
H_{*}=\left[\begin{array}{llllllll}
1 & 0 & 0 & 0 & 0 & 0 & 0 & 0 \\
0 & 1 & 0 & 0 & 0 & 0 & 0 & 0
\end{array}\right] .
\end{gathered}
$$

\subsection{The observers}

In this section, EAOB and Nisocia observer for a 2 DOF nonlinear teleoperation system are presented.

\subsubsection{EAOB}

According to Section 3.2, by defining

$$
\hat{X}_{*}=\left[\begin{array}{c}
\hat{q}_{* 1} \\
\hat{q}_{* 2} \\
\hat{q}_{* 1} \\
\hat{\vec{q}}_{* 2} \\
\hat{f}_{h / e 1} \\
\hat{f}_{h / e 2} \\
\hat{\theta}_{* 1} \\
\hat{\theta}_{* 2}
\end{array}\right],
$$

the $\operatorname{EAOB}(S=1)$ is given by

$$
\dot{\hat{X}}_{*}=f_{*}\left(\hat{X}_{*}, T_{*}\right)+P_{*} H_{*}^{T} R_{*}^{-1}\left(Y_{*}-H_{*} \hat{X}_{*}\right),
$$

where 


$$
\dot{P}_{*}=\frac{\partial f_{*}}{\partial \grave{X}_{*}} P_{*}+P_{*} \frac{\partial f_{*}^{T}}{\partial \grave{X}_{*}}+G_{*} Q_{*} G_{*}^{T}-P_{*} H_{*}^{T} R_{*}^{-1} H_{*} P_{*}
$$

where $Q_{*}$ and $R_{*}$ are variance matrices of the process noise $\xi_{*}$ and the measurement noise $\eta_{*}$, respectively, and $P_{*}$ represents the estimate of the variance of error of the system states' estimate, and

$$
F_{*}=\frac{\partial f_{*}}{\partial \hat{X}_{*}}=\left[\begin{array}{cccccccccc}
0 & 0 & & 1 & 0 & 0 & 0 & 0 & 0 & \\
0 & 0 & & 0 & 1 & 0 & 0 & 0 & 0 & \\
F_{* 31} & F_{* 32} & F_{* 33} & F_{* 34} & F_{* 35} & F_{* 36} & F_{* 37} & F_{* 38} \\
F_{* 41} & F_{* 42} & F_{* 43} & F_{* 44} & F_{* 45} & F_{* 46} & F_{* 47} & F_{* 48} \\
0 & 0 & & 0 & 0 & 0 & 0 & 0 & 0 & \\
0 & 0 & & 0 & 0 & 0 & 0 & 0 & 0 & \\
0 & 0 & & 0 & 0 & 0 & 0 & 0 & 0 & \\
0 & 0 & & 0 & 0 & 0 & 0 & 0 & 0 &
\end{array}\right],
$$

where

$$
\begin{gathered}
{\left[\begin{array}{ll}
F_{* 31} & F_{* 32} \\
F_{* 41} & F_{* 42}
\end{array}\right]=-M_{*}^{-1}\left(\frac{\partial M_{*}}{\partial \hat{q}_{*}} \ddot{\hat{q}}_{*}+\frac{\partial V_{*} \dot{q}_{*}}{\partial \hat{q}_{*}} \mp \frac{\partial J_{*}^{T}\left(\hat{q}_{*}\right) \hat{f}_{h / e}}{\partial \hat{q}_{*}}\right),} \\
{\left[\begin{array}{ll}
F_{* 33} & F_{* 34} \\
F_{* 43} & F_{* 44}
\end{array}\right]=-M_{*}^{-1} \frac{\partial V_{*} \dot{\hat{q}}_{*}}{\partial \hat{q}_{*}}} \\
{\left[\begin{array}{ll}
F_{* 35} & F_{* 36} \\
F_{* 45} & F_{* 46}
\end{array}\right]=\mp M_{*}^{-1} J_{*}^{T}\left(\hat{q}_{*}\right)} \\
{\left[\begin{array}{ll}
F_{* 37} & F_{* 38} \\
F_{* 47} & F_{* 48}
\end{array}\right]=-M_{*}^{-1}\left(\frac{\partial M_{*}}{\partial \hat{\theta}_{*}} \ddot{\hat{q}}_{*}+\frac{\partial V_{*} \dot{q}_{*}}{\partial \hat{\theta}_{*}}\right)}
\end{gathered}
$$

\subsubsection{Nisocia observer}

By defining

$$
X_{* 1}=\left[\begin{array}{c}
q_{* 1} \\
q_{* 2}
\end{array}\right], X_{* 2}=\left[\begin{array}{c}
\dot{q}_{* 1} \\
\dot{q}_{* 2}
\end{array}\right]
$$

the Nicosia observer ${ }^{6}$ for the master or slave side of a 2 DOF teleoperation system is formulated as follows,

$$
\begin{aligned}
& \dot{\hat{X}}_{* 1}=\hat{X}_{* 2}+K_{* 1}\left(X_{* 1}-\hat{X}_{* 1}\right) . \\
& \dot{\hat{X}}_{* 2}=M_{*}^{-1}\left(-V_{*} \hat{X}_{* 2}+T_{*}+K_{* 2}\left(X_{* 1}-\hat{X}_{* 1}\right)\right) .
\end{aligned}
$$

Thus, the estimated forces are written as 


$$
\hat{f}_{h / e}=\left[\begin{array}{l}
\hat{f}_{h / e 1} \\
\hat{f}_{h / e 2}
\end{array}\right]= \pm J_{*}^{T^{-1}} K_{* 2}\left(X_{* 1}-\hat{X}_{* 1}\right)
$$

where $K_{* 1}$ and $K_{* 2}$ are symmetric positive definite matrices.

\subsection{Simulation}

In this Section, the performance of EAOB and Nicosia observer based control schemes in teleoperation systems are studies and compared through computer simulation.

\subsubsection{Initial conditions}

In order to compare EAOB and Nicosia observer algorithms, their parameters including controller feedback gains, desired operator rendered force, the environment rendered force, and pre-filter for desired operator force are kept the same. According to guidelines well justified theoretically in Section 3 for implementation of the proposed teleoperation scheme, the choice of design parameters is made as follows.

The operator rendered forces $f_{h 1}, f_{h 2}$ in $Z_{m 1}$ and $Z_{m 2}$ directions are, respectively assumed to be a step signal with a peak value of $2 \mathrm{~N}$, which is pre-filtered with a critically damped second order linear filter with a bandwidth of $2 \mathrm{rad} / \mathrm{s}$ and a transfer function of $\frac{4}{s^{2}+4 s+4}$, while the environment rendered forces in $Z_{s 1}$ and $Z_{s 2}$ directions are, respectively assumed to be

$$
f_{e 1}=5 *\left(Z_{s 1}-1.0\right), f_{e 2}=5 *\left(Z_{s 2}-1.0\right) \text {. }
$$

The sampling period $T_{\mathrm{s}}$ is set to 0.001 seconds. The initial angels and angular velocities of the joints of the master and slave robot manipulators are set to zero:

$q_{* 1-\text { initial }}=0, q_{* 2-\text { initial }}=0, \dot{q}_{* 1 \text {-initial }}=0, \dot{q}_{* 2 \text {-initial }}=0,(*=\mathrm{m} / \mathrm{s})$ implying that robot manipulators are initially at rest. The actual inertial parameters for the master and slave robot manipulators are chosen identically as $\theta_{* 1}=1.0, \theta_{* 2}=2.0 ; l_{* 1}=l_{* 2}=1.0$.

The force controller gains of both approaches for the master system without measurement noise, are $K_{m c}=$

$\left[\begin{array}{cc}0.5 & 0 \\ 0 & 0.5\end{array}\right], K_{m i c}=\left[\begin{array}{cc}1.4 & 0 \\ 0 & 1.4\end{array}\right], K_{m d}=\left[\begin{array}{cc}200 & 0 \\ 0 & 200\end{array}\right]$, and $K_{m p}=\left[\begin{array}{cc}5000 & 0 \\ 0 & 5000\end{array}\right]$, while $K_{m c}=\left[\begin{array}{cc}2.0 & 0 \\ 0 & 2.0\end{array}\right]$, $K_{m i c}=\left[\begin{array}{cc}1.4 & 0 \\ 0 & 1.4\end{array}\right], K_{m d}=\left[\begin{array}{cc}200 & 0 \\ 0 & 200\end{array}\right]$, and $K_{m p}=\left[\begin{array}{cc}5000 & 0 \\ 0 & 5000\end{array}\right]$ for the master system considering the measurement noise. 
The position controller gains of both approaches for the slave system are $K_{s d}=\left[\begin{array}{cc}40 & 0 \\ 0 & 40\end{array}\right]$, and $K_{s p}=$ $\left[\begin{array}{cc}400 & 0 \\ 0 & 400\end{array}\right]$, despite the presence of measurement noise.

The specific initial conditions for EAOB based control scheme are chosen as follows:

The initial conditions for EAOB at the master and slave sides when there is no disturbance in the entire teleoperation system are

$$
\begin{gathered}
R_{m}=\left[\begin{array}{cc}
1.0 e-7 & 0 \\
0 & 1.0 e-7
\end{array}\right], \\
Q_{m}=\operatorname{diag}\{1.0 e-10,1.0 e-10,1.0 e-10,1.0 e-10,0.3,0.3,0.025,0.025\}, \\
P_{0 m}=\operatorname{diag}\{1.0 e-4,1.0 e-4,1.0 e-4,1.0 e-4,1.0,1.0,1.0 e-2,1.0 e-2\} ; \\
R_{s}=\left[\begin{array}{cc}
1.0 e-7 & 0 \\
0 & 1.0 e-7
\end{array}\right], \\
Q_{s}=\operatorname{diag}\{1.0 e-10,1.0 e-10,1.0 e-10,1.0 e-10,5.0,5.0,3.0,3.0\}, \\
P_{0 s}=\operatorname{diag}\{1.0 e-4,1.0 e-4,1.0 e-4,1.0 e-4,5.0,5.0,5.0,5.0\} .
\end{gathered}
$$

The initial conditions for EAOB at the master and slave sides when only inertial parameter variations (20\% of the actual parameters) exist in both master and slave sides of the whole system are

$$
\begin{gathered}
R_{m}=\left[\begin{array}{cc}
1.0 e-7 & 0 \\
0 & 1.0 e-7
\end{array}\right], \\
Q_{m}=\operatorname{diag}\{1.0 e-10,1.0 e-10,1.0 e-10,1.0 e-10,0.3,0.3,0.025,0.025\}, \\
P_{0 m}=\operatorname{diag}\{1.0 e-4,1.0 e-4,1.0 e-4,1.0 e-4,1.0,1.0,1.0 e-2,1.0 e-2\} ; \\
R_{s}=\left[\begin{array}{cc}
1.0 e-7 & 0 \\
0 & 1.0 e-7
\end{array}\right], \\
Q_{s}=\operatorname{diag}\{1.0 e-10,1.0 e-10,1.0 e-10,1.0 e-10,1.0,1.0,0.08,0.7\}, \\
P_{0 s}=\operatorname{diag}\{1.0 e-4,1.0 e-4,1.0 e-4,1.0 e-4,1.0,1.0,1.0,1.0\} .
\end{gathered}
$$

The initial conditions for EAOB at the master and slave sides when only measurement noises $(N \sim(0,1.0 \mathrm{e}-5))$ are considered in both master and slave sides of the teleoperation system are 


$$
\begin{gathered}
R_{m}=\left[\begin{array}{cc}
1.0 e-5 & 0 \\
0 & 1.0 e-5
\end{array}\right], \\
Q_{m}=\operatorname{diag}\{1.0 e-10,1.0 e-10,1.0 e-10,1.0 e-10,0.3,0.3,0.025,0.025\}, \\
P_{0 m}=\operatorname{diag}\{1.0 e-4,1.0 e-4,1.0 e-4,1.0 e-4,1.0,1.0,1.0 e-2,1.0 e-2\} ; \\
R_{s}=\left[\begin{array}{cc}
1.0 e-5 & 0 \\
0 & 1.0 e-5
\end{array}\right], \\
Q_{s}=\operatorname{diag}\{1.0 e-10,1.0 e-10,1.0 e-10,1.0 e-10,0.55,0.5,0.08,0.7\}, \\
P_{0 s}=\operatorname{diag}\{1.0 e-4,1.0 e-4,1.0 e-4,1.0 e-4,1.0,1.0,1.0,1.0\} .
\end{gathered}
$$

The initial conditions for EAOB at the master and slave sides when both measurement noises $(N \sim(0,1.0 \mathrm{e}-5))$ and inertial parameter variations ( $20 \%$ of actual parameters) are considered in both master and slave sides of the teleoperation system are

$$
\begin{gathered}
R_{m}=\left[\begin{array}{cc}
1.0 e-5 & 0 \\
0 & 1.0 e-5
\end{array}\right], \\
Q_{m}=\operatorname{diag}\{1.0 e-10,1.0 e-10,1.0 e-10,1.0 e-10,0.3,0.3,0.025,0.025\}, \\
P_{0 m}=\operatorname{diag}\{1.0 e-4,1.0 e-4,1.0 e-4,1.0 e-4,1.0,1.0,1.0 e-2,1.0 e-2\} ; \\
R_{s}=\left[\begin{array}{cc}
1.0 e-5 & 0 \\
0 & 1.0 e-5
\end{array}\right], \\
Q_{s}=\operatorname{diag}\{1.0 e-10,1.0 e-10,1.0 e-10,1.0 e-10,0.55,0.55,0.08,0.7\}, \\
P_{0 s}=\operatorname{diag}\{1.0 e-4,1.0 e-4,1.0 e-4,1.0 e-4,1.0,1.0,1.0,1.0\} .
\end{gathered}
$$

In all situations, the specific observer gains for Nicosia observer based control scheme are chosen as follows:

By trial and error, the observer gains for Nicosia observer on the master end are chosen as $K_{m 1}=\left[\begin{array}{cc}20 & 0 \\ 0 & 20\end{array}\right]$, and $K_{m 2}=\left[\begin{array}{cc}200 & 0 \\ 0 & 200\end{array}\right]$

By trial and error, the observer gains for Nicosia observer on the master end are chosen as $K_{S 1}=\left[\begin{array}{cc}20 & 0 \\ 0 & 20\end{array}\right]$, and $K_{s 2}=\left[\begin{array}{cc}200 & 0 \\ 0 & 200\end{array}\right]$ 


\subsubsection{Simulation results}

The results for EAOB based control scheme under different noise conditions and parameter variations are illustrated in Fig. 2-9, whereas for Nicosia observer based control scheme under the same conditions are shown in Fig. 10-17.
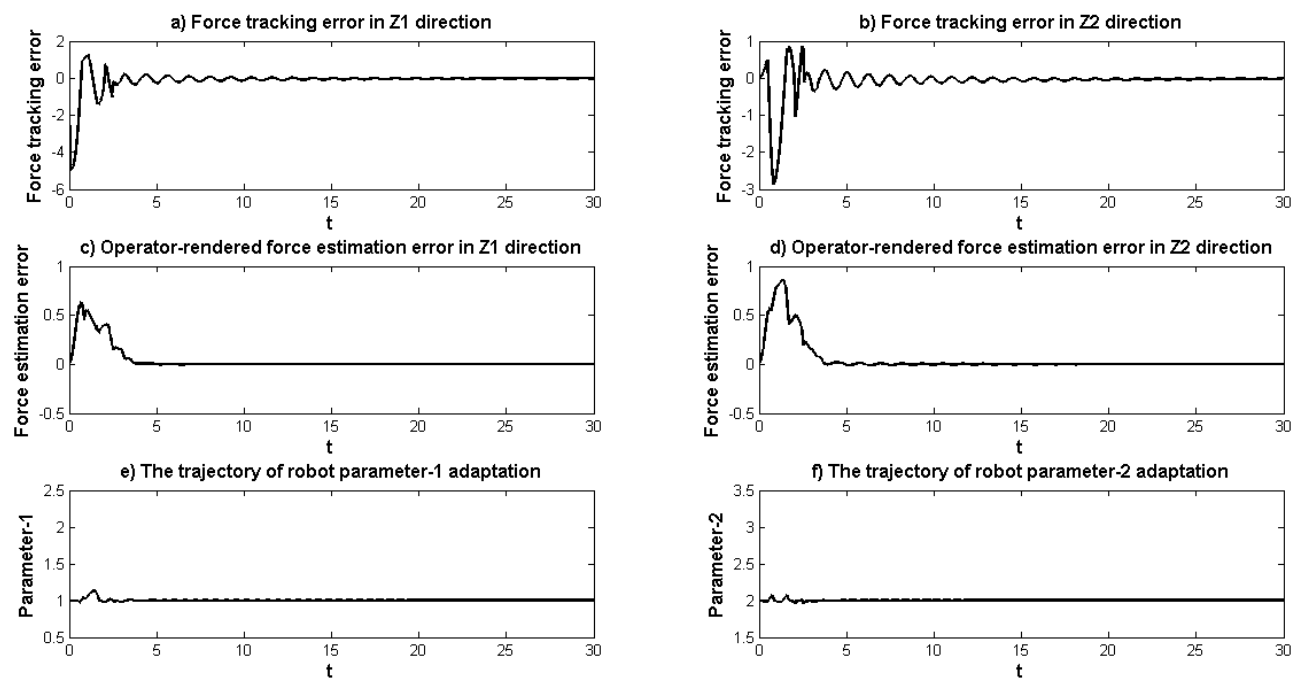

Fig.2. The master side performance of EAOB based approach without measurement noise and parameter variation.
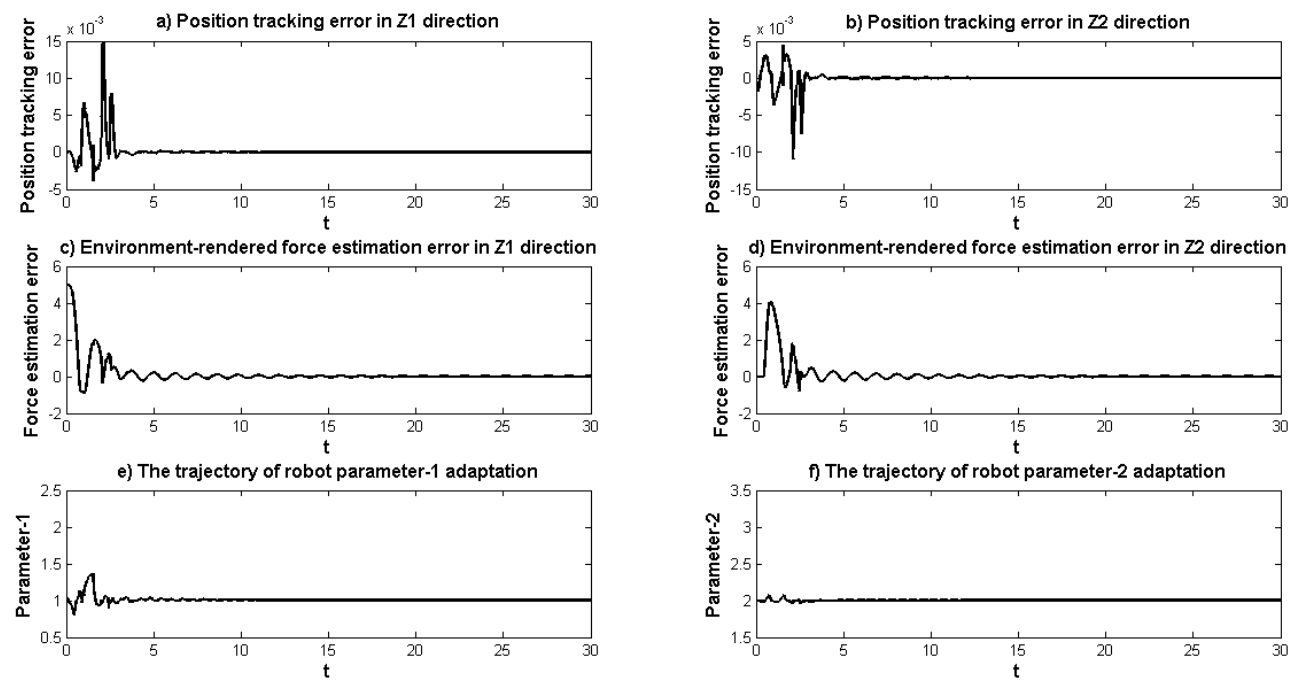

Fig.3. The slave side performance of EAOB based approach without measurement noise and parameter variation. 

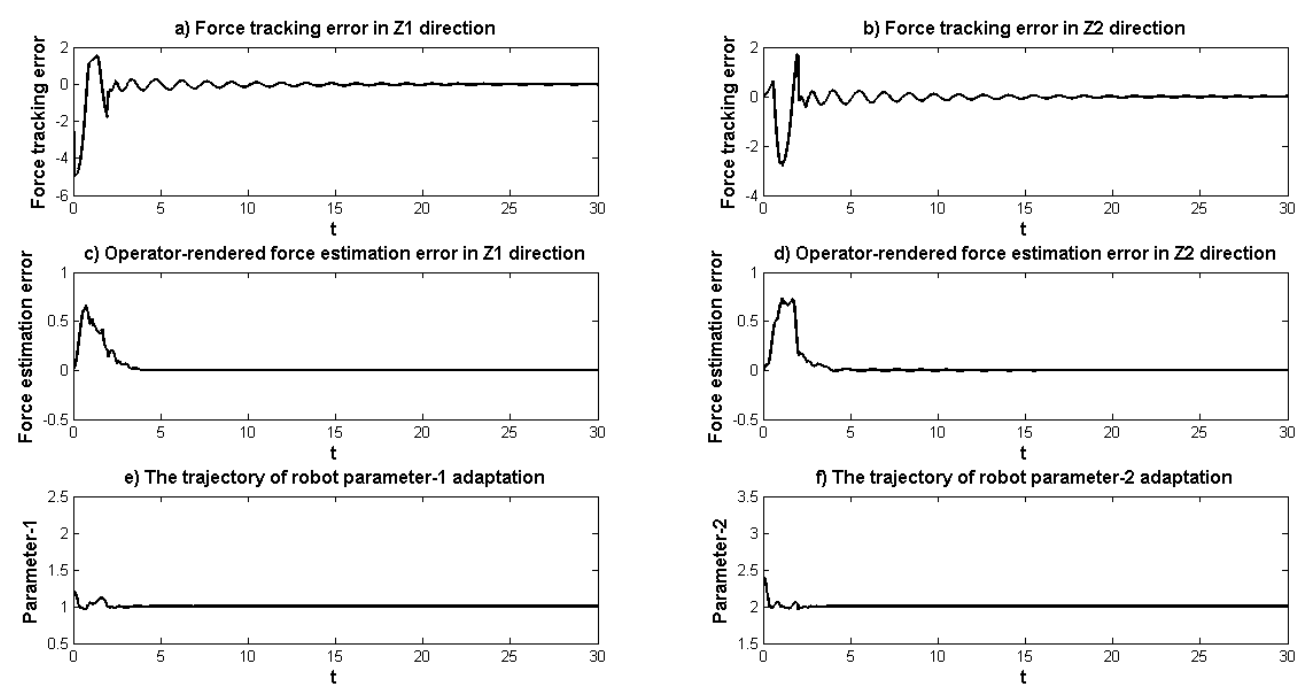

Fig.4. The master side performance of EOAB based approach with only parameter variation: $20 \%$ of actual parameters.
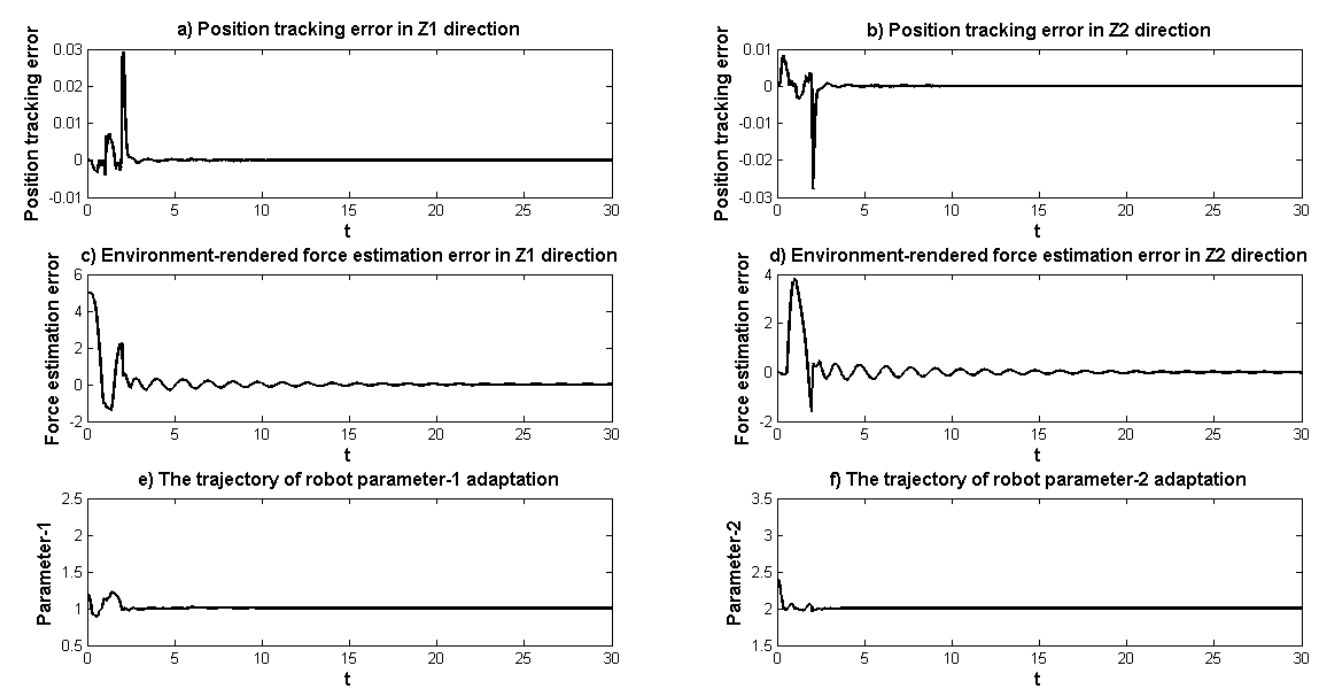

Fig.5. The slave side performance of EOAB based approach with only parameter variation: $20 \%$ of actual parameters. 

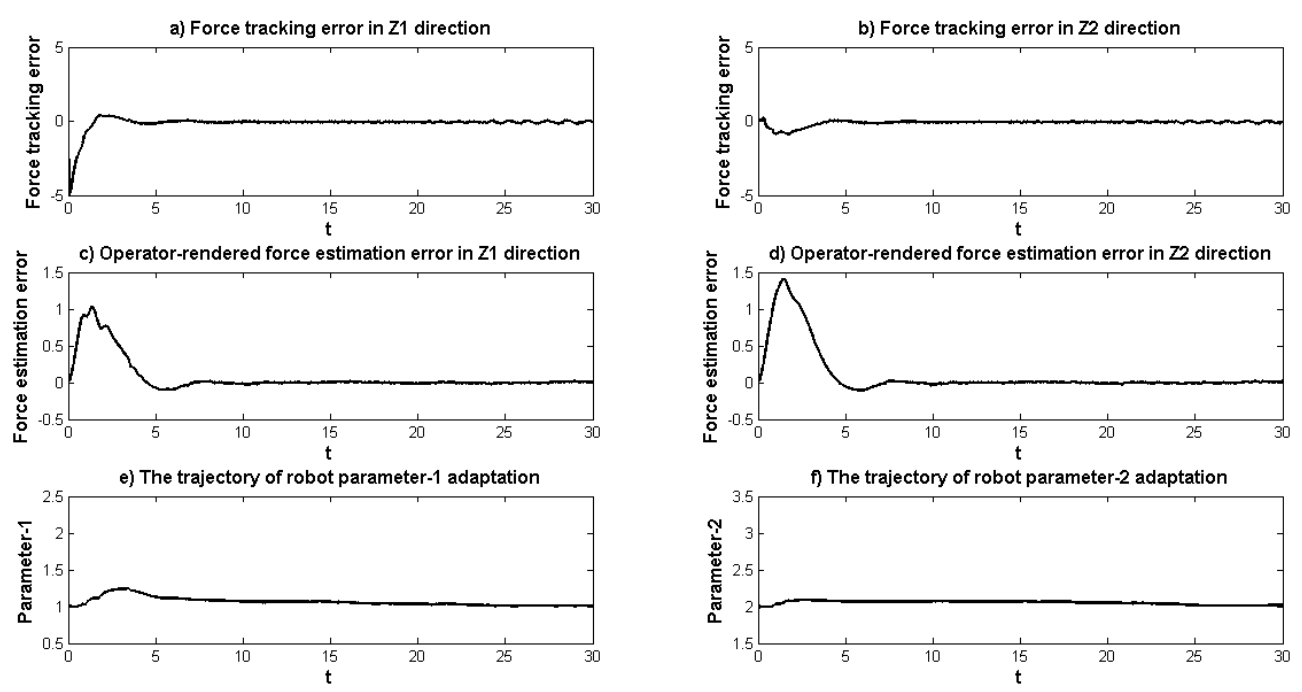

Fig.6. The master side performance of EOAB based approach with only measurement noise: $N \sim(0,1.0 \mathrm{e}-5)$.
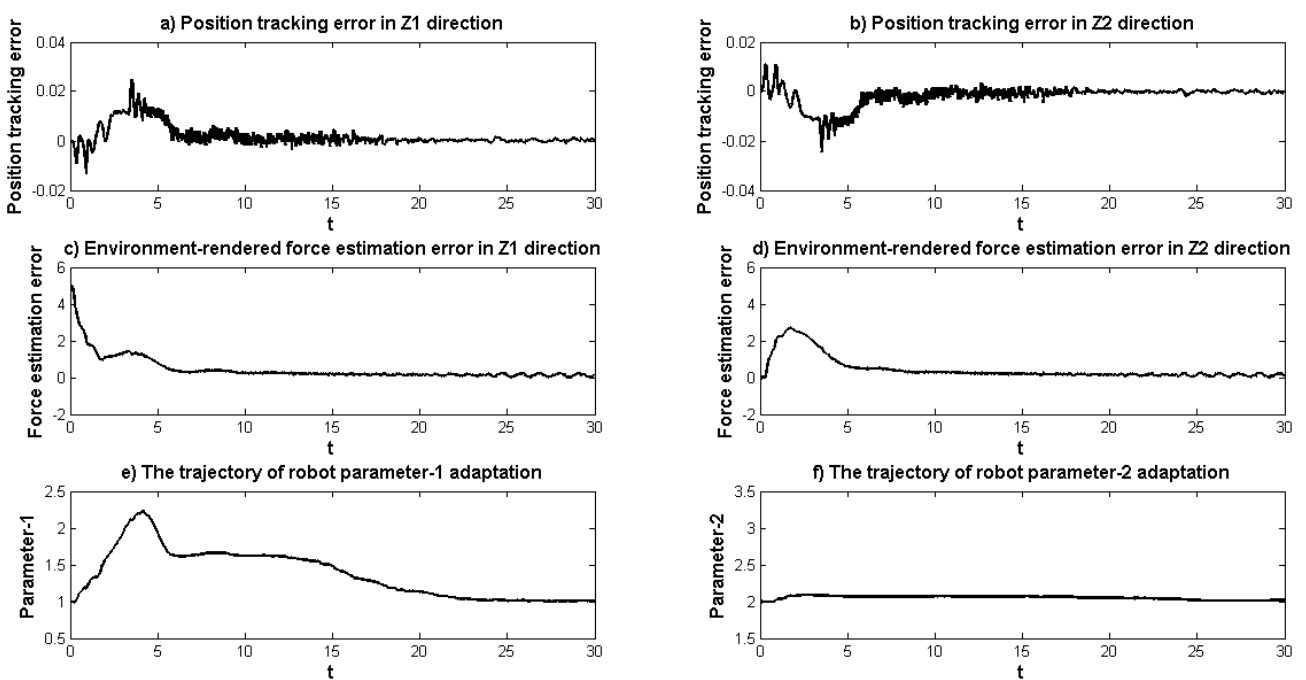

Fig.7. The slave side performance of EAOB based approach with measurement noise: $N \sim(0,1.0 \mathrm{e}-5)$. 

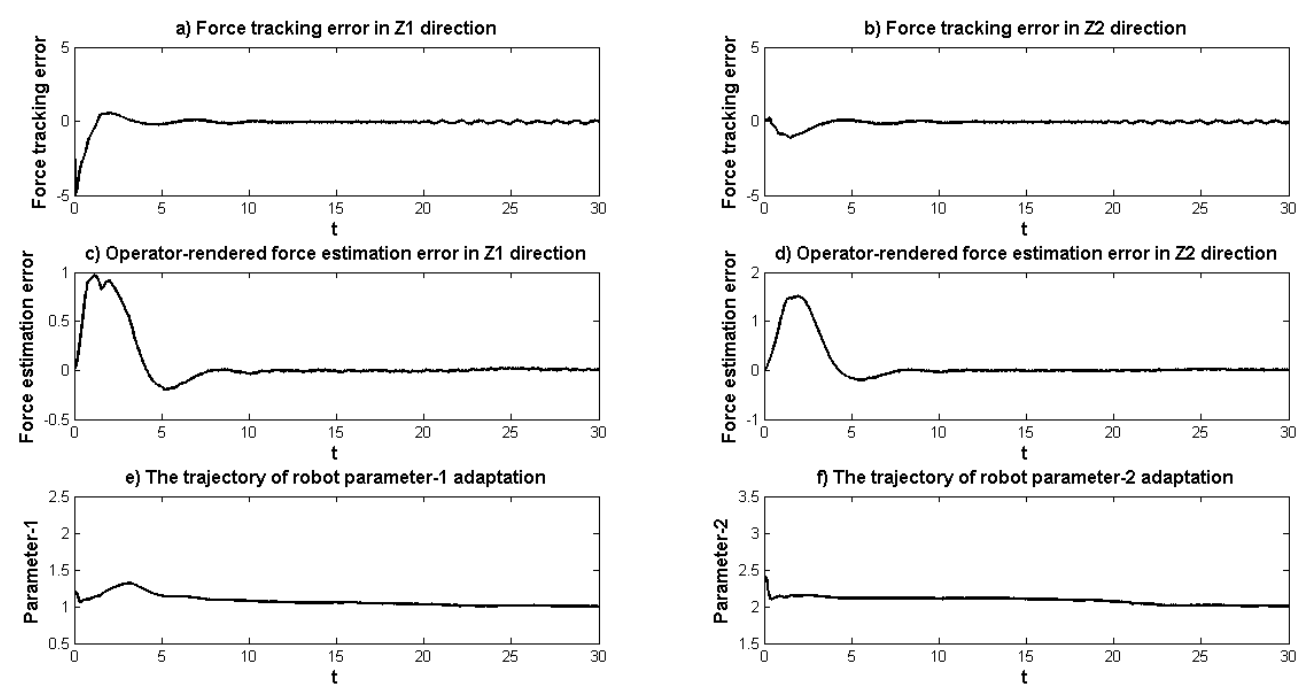

Fig.8. The master side performance of EAOB based approach with measurement noise: $N \sim(0,1.0 \mathrm{e}-5)$ and parameter variation: $20 \%$ of actual parameters.
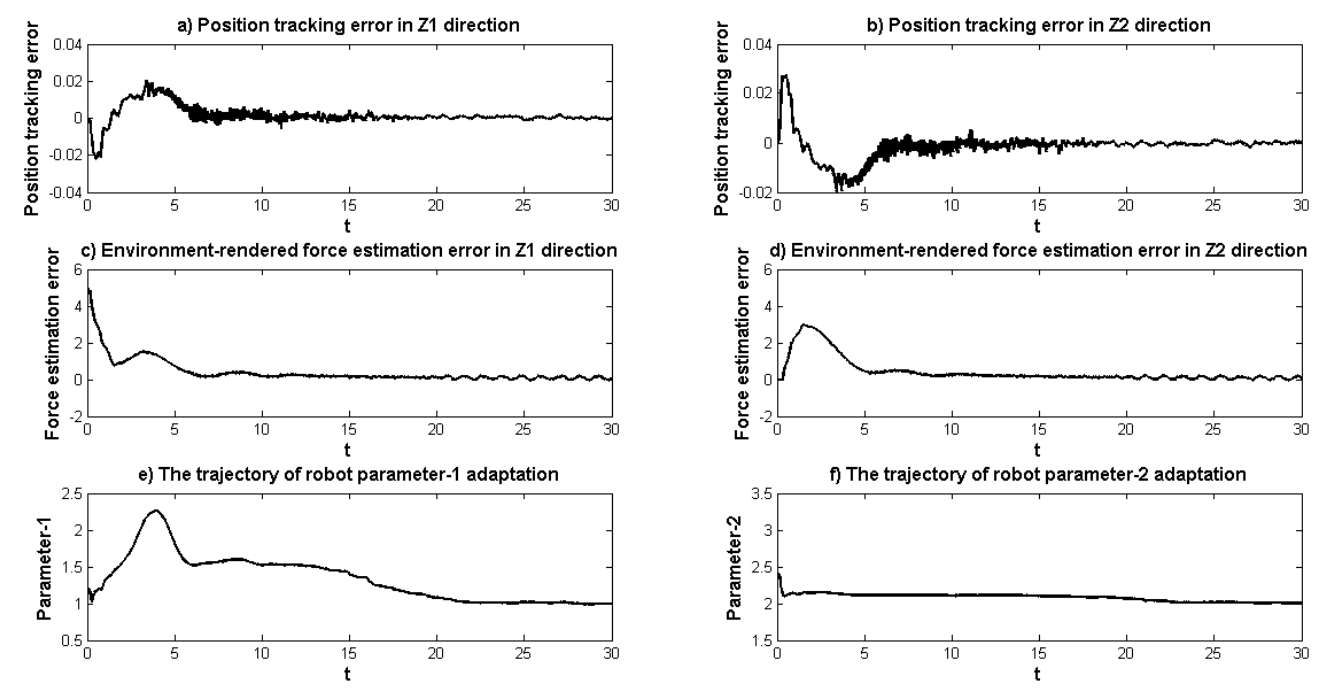

Fig.9. The slave side performance of EAOB based approach with measurement noise: $N \sim(0,1.0 \mathrm{e}-5)$ and parameter variation: $20 \%$ of actual parameters.

Fig. 2-9 show simulation results of EAOB based approach under different situations, and demonstrate good performances of EAOB based approach in the presence of different measurement noises and parameter variations. Fig. 2,4,6,8 depict the master side performance of EAOB based approach, while Fig. 3,5,7,9 show the slave side performance of EAOB based approach. Specifically, Fig. i.a), Fig. i.c), and Fig. i.e) $(i=2,4,6,8)$ show the trajectories of the error between desired operator force and estimated environment force in $Z_{m 1}$ direction, the error between actual and estimated operator force in $Z_{m 1}$ direction, and parameter-1 of the master 
robot manipulator, while Fig. i.b), Fig. i.d), and Fig. i.f) $(i=2,4,6,8)$ provide the trajectories of the error between desired operator force and estimated environment force in $Z_{s 2}$ direction, and the error between actual and estimated operator force in $Z_{s 2}$ direction, and parameter-2 of the master robot manipulator. Meanwhile, Fig. j.a), Fig. j.c), and Fig. j.e) (j=3,5,7,9) show the trajectories of the error between actual position of the master and slave robot manipulators in $Z_{m 1}$ direction, the error between actual and estimated environment force in $Z_{m 1}$ direction, and parameter-1 of the slave robot manipulator, while Fig. j.b), Fig. j.d), and Fig. j.f) (j=3,5,7,9) provide the trajectories of the error between actual position of the master and slave robot manipulators in $Z_{s 2}$ direction, the error between actual and estimated environment force in $Z_{s 2}$ direction, and parameter-2 of the slave robot manipulator.

In Fig.2 and Fig. 3, the simulation results are obtained in the absence of both robot parameter variations and measurement noises at both the master and slave sides of the teleoperation system. As observed in Fig.2, on the master end, the desired operator forces rendered in $Z_{m 1}$ and $Z_{m 2}$ directions are accurately tracked by the environment forces rendered in $Z_{m 1}$ and $Z_{m 2}$ directions, and the operator force estimations are also accurate. The trajectories of master robot parameter- 1 and parameter- 2 adaptation almost remain at 1.0 and 2.0, respectively. Similarly, on the slave end, as depicted in Fig. 3, positions of the master robot manipulator in $Z_{s 1}$ and $Z_{s 2}$ directions are accurately tracked by the positions of the slave robot manipulator in $Z_{s 1}$ and $Z_{s 2}$ directions respectively, and the environment force estimations are also accurate. The trajectories of slave robot parameter-1 and parameter-2 adaptation almost remain at 1.0 and 2.0 , respectively as there is no parameter variation in the system.

Fig. 4 and Fig. 5 depict the performance of the EAOB based approach only with parameter variation: $20 \%$ of the actual parameters at both the master and slave sides of the teleoperation system. In this situation, at the master side, as depicted in Fig. 4, the trajectories of force tracking in $Z_{m 1}$ and $Z_{m 2}$ directions are still accurate, the operator force estimation is still good, and the trajectories of master robot parameter- 1 and parameter-2 converge from $(1.2,2.4)$ to $(1.0,2.0)$, respectively. Meanwhile, In Fig. 5, the trajectories of position tracking in $Z_{s 1}$ and $Z_{s 2}$ directions at the slave side are also accurate, the environment force estimation is still satisfactory, and the trajectories of slave robot parameter- 1 and parameter- 2 converge from $(1.2,2.4)$ to $(1.0,2.0)$, respectively.

Fig. 6 and Fig. 7 provide the performance of the EAOB based approach when only measurement noises are considered in the teleoperation system. As shown in Fig. 6 and Fig. 7, it is easy to observe that when Gaussian 
noise $(N \sim(0,1.0 \mathrm{e}-5))$ are added at both the master and slave sides, the trajectories of force tracking in $Z_{m 1}$ and $Z_{m 2}$ directions at the master side and position tracking in $Z_{s 1}$ and $Z_{s 2}$ directions at the slave side are still good, while the accuracy of the operator and environment force estimations is still acceptable. The trajectories of the master and slave robot parameter-1 and parameter-2 adaptation almost remain at 1.0 and 2.0, respectively.

In Fig. 8 and Fig. 9, the performance of the EAOB based approach with measurement noise: $N \sim(0,1.0 \mathrm{e}-5)$ and parameter variation: $20 \%$ of the actual parameters at both the master and slave sides of the teleoperation system is examined. From Fig. 8, it is easy to see that the trajectories of force tracking in $Z_{m 1}$ and $Z_{m 2}$ directions on the master end remain accurate, and the operator force estimation still remains satisfactory. Furthermore, the trajectories of master robot parameter-1 and parameter-2 adaptation converge from $(1.2,2.4)$ to $(1.0,2.0)$, respectively. Meanwhile, In Fig. 9, the trajectories of position tracking in $Z_{s 1}$ and $Z_{s 2}$ directions on the slave end remain accurate, the environment force estimation still maintains satisfactory, and the trajectories of slave robot parameter-1 and parameter-2 adaptation converge from $(1.2,2.4)$ to $(1.0,2.0)$, respectively.
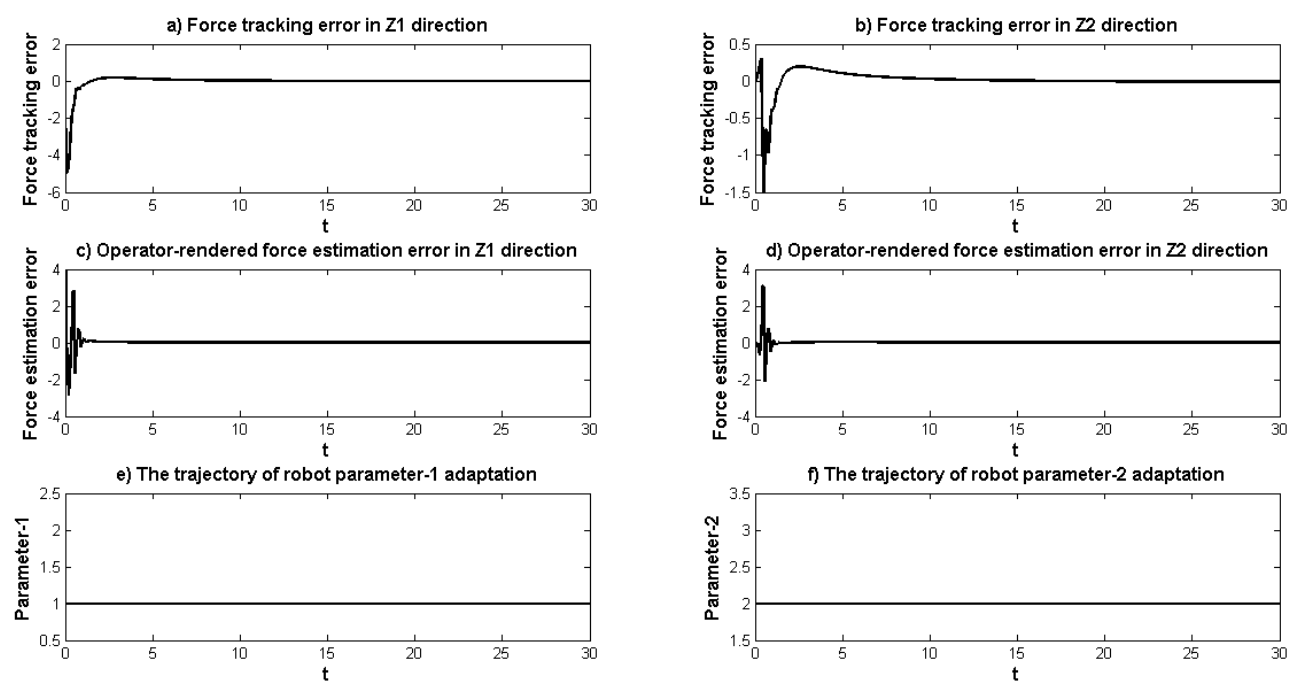

Fig. 10. The master side performance of Nicosia observer based approach without measurement noise and parameter variation. 

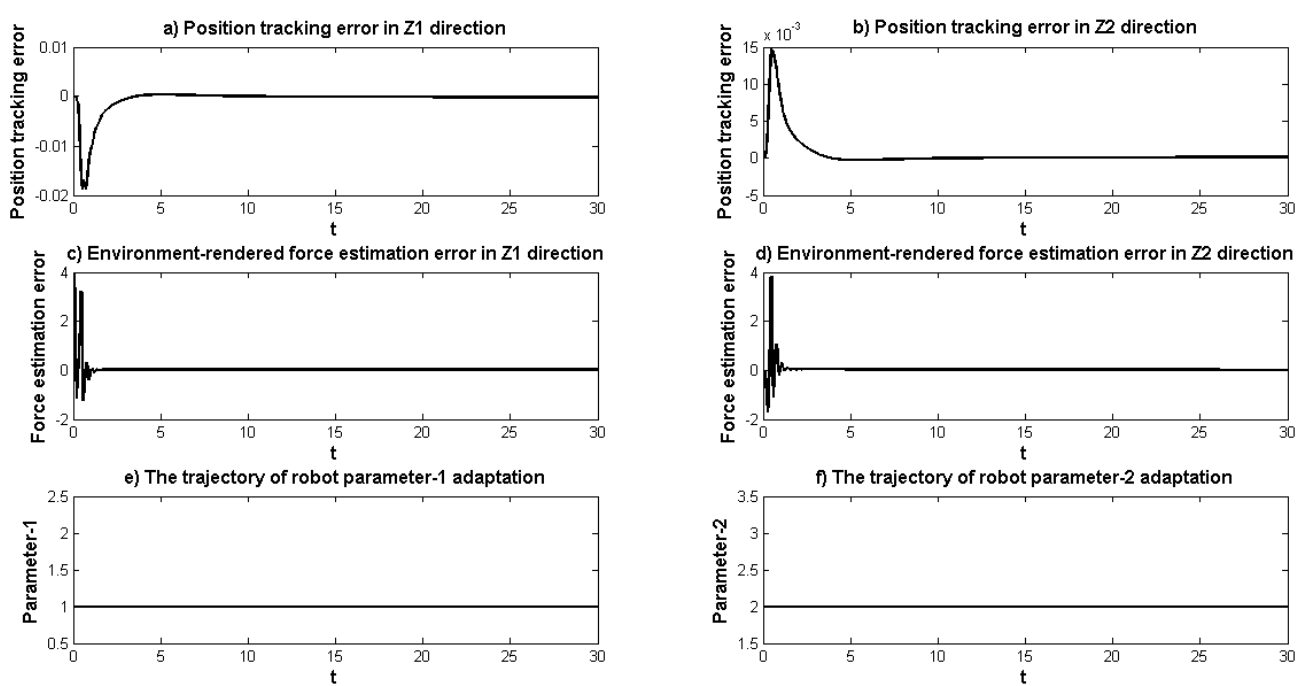

Fig.11. The slave side performance of Nicosia observer based approach without measurement noise and parameter variation.
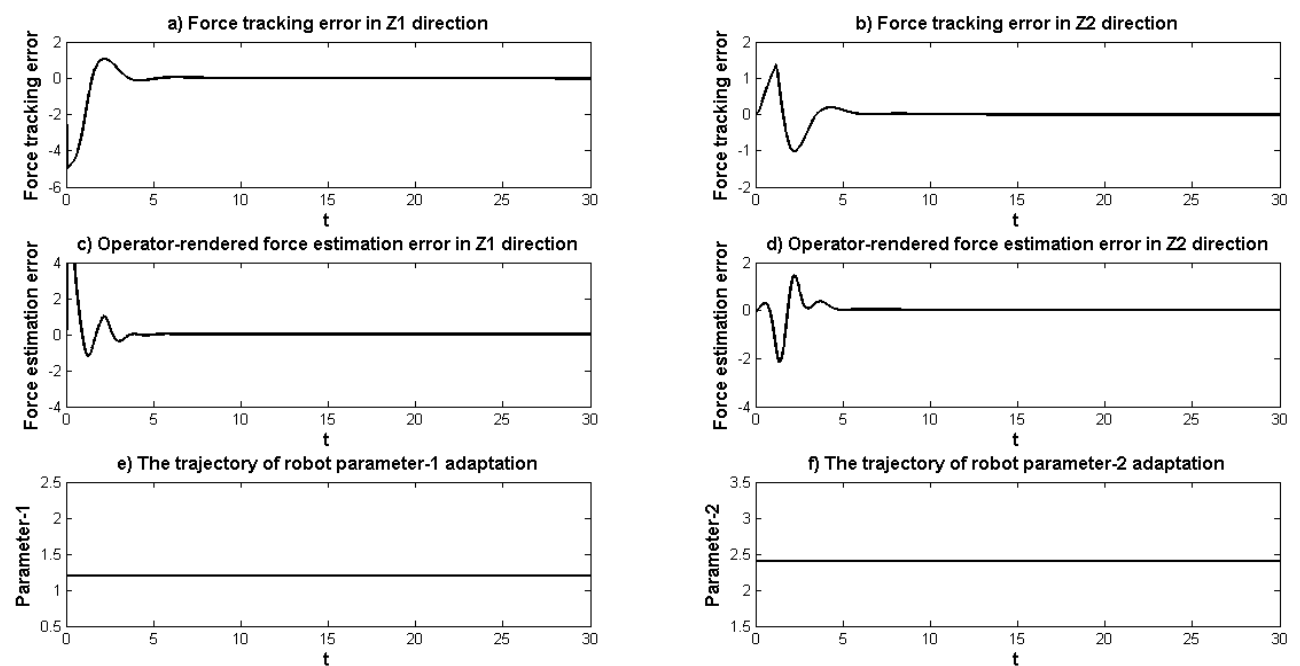

Fig.12. The master side performance of Nicosia observer based approach with only parameter variation: $20 \%$ of actual parameters. 

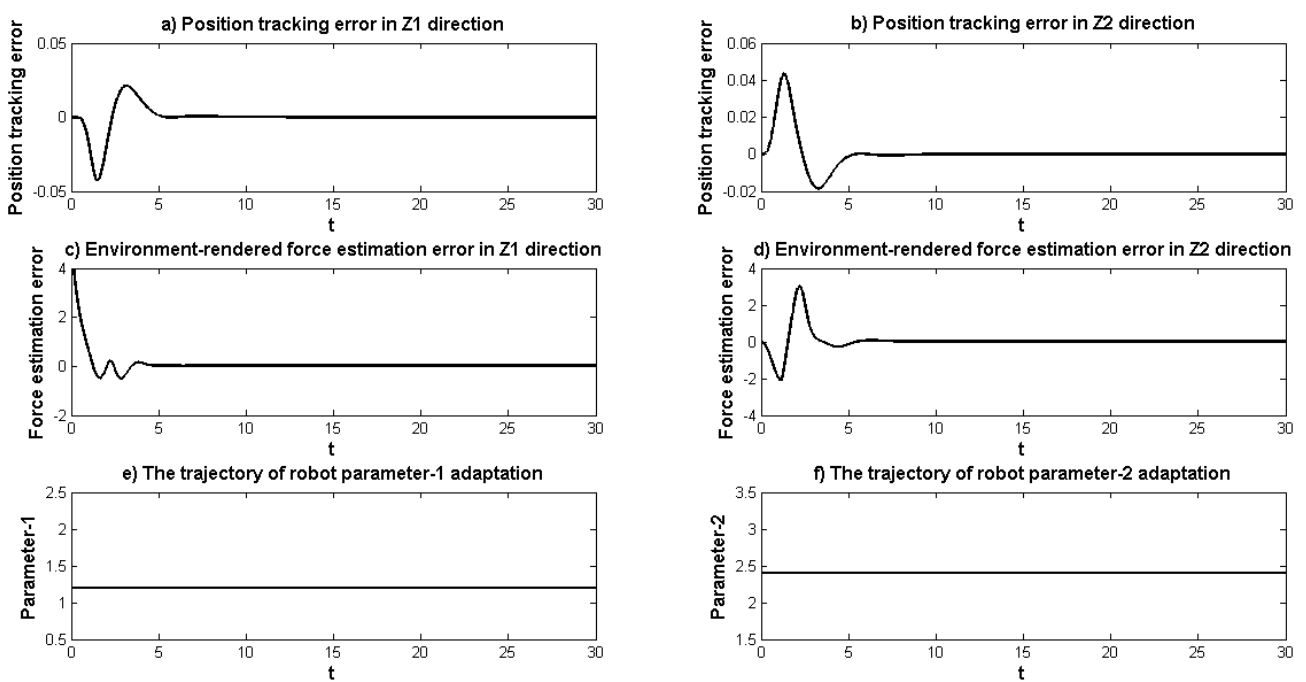

Fig.13. The slave side performance of Nicosia observer based approach with only parameter variation: $20 \%$ of actual parameters.
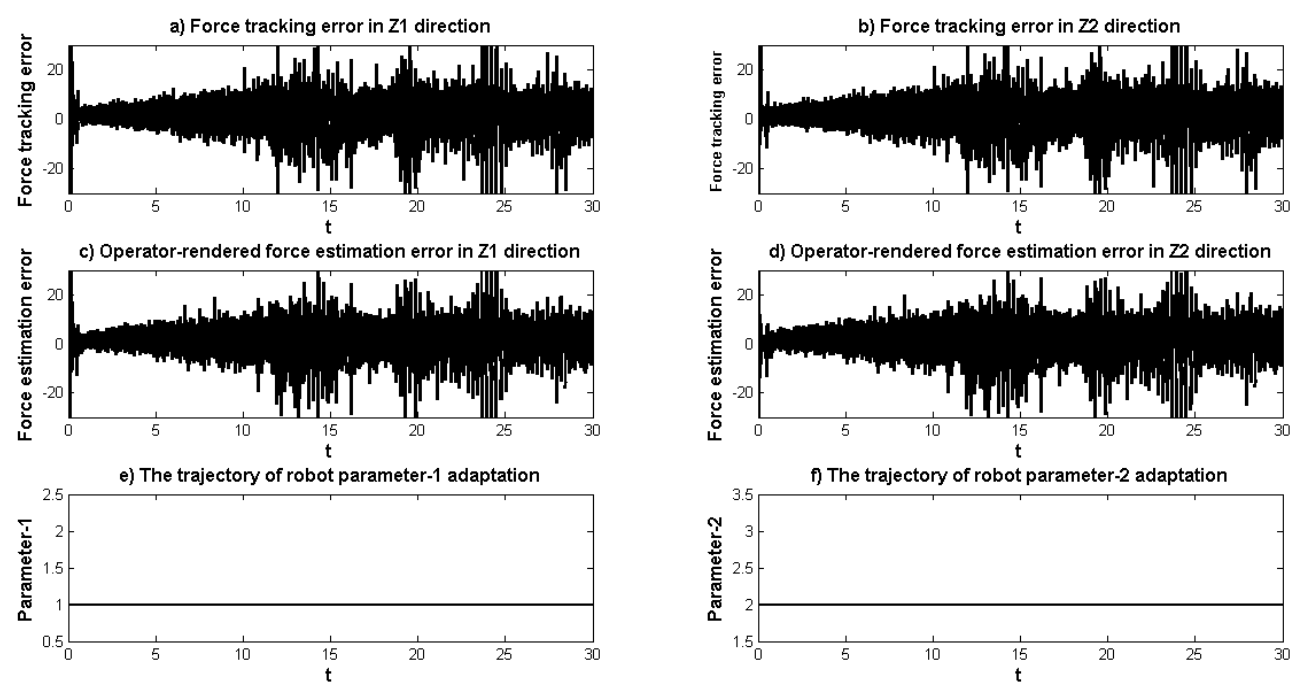

Fig.14. The master side performance of Nicosia observer based approach with only measurement noise: $N \sim(0,1.0 \mathrm{e}-5)$. 

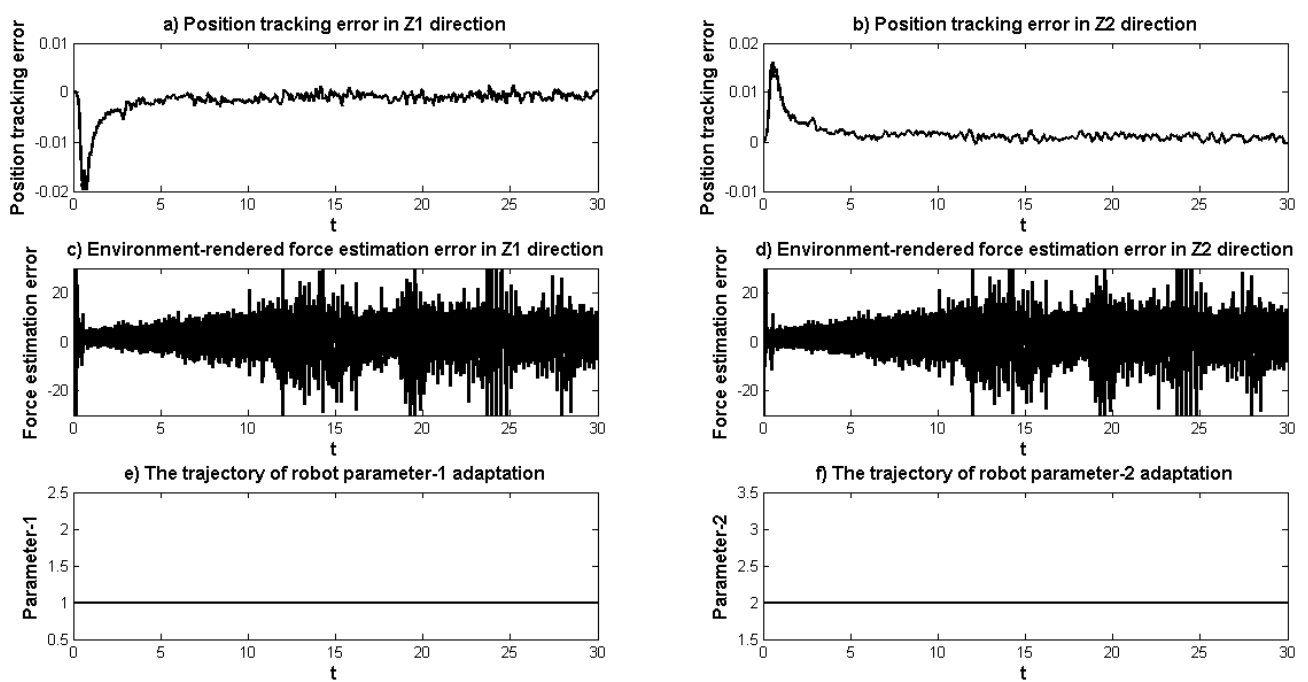

Fig.15. The slave side performance of Nicosia observer based approach with only measurement noise: $N \sim(0,1.0 \mathrm{e}-5)$.
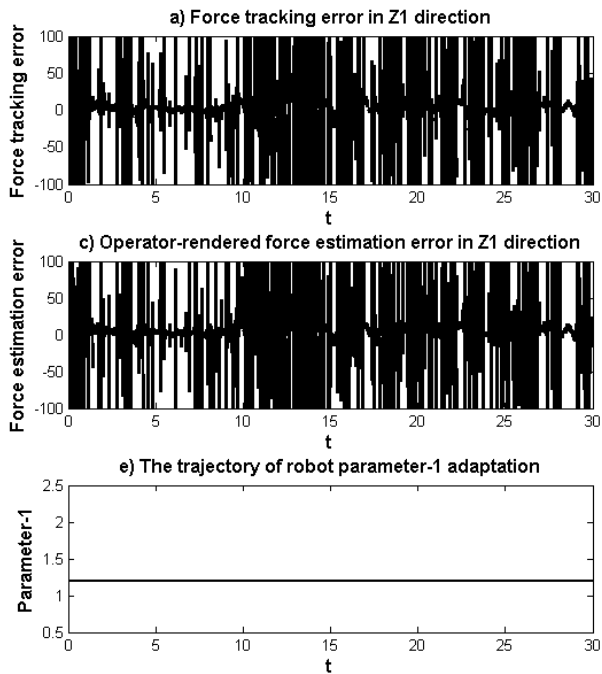

b) Force tracking error in $\mathbf{Z 2}$ direction
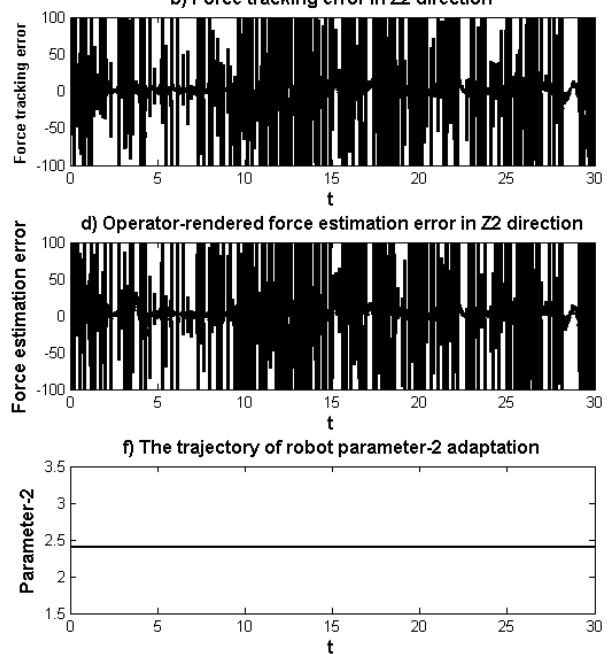

Fig.16. The master side performance of Nicosia observer based approach with measurement noise: $N \sim(0,1.0 \mathrm{e}-5)$ and parameter variation: $20 \%$ of actual parameters. 

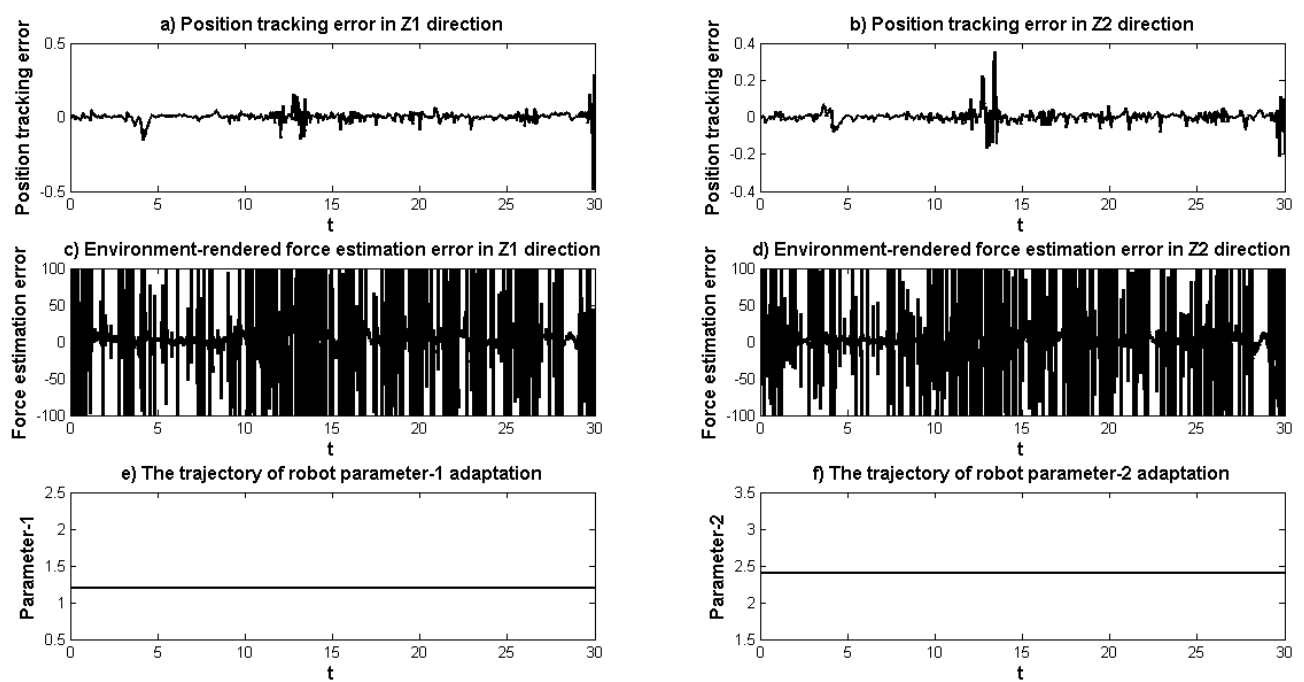

Fig.17. The slave side performance of Nicosia observer based approach with measurement noise: $N \sim(0,1.0 \mathrm{e}-5)$ and parameter variation: $20 \%$ of actual parameters.

Fig. 10-17 illustrate simulation results of the Nisocia observer based approach under different situations, and show that the performances of Nisocia observer based approach deteriorates when the effects of measurement noise and parameter variation are taken into account. Fig. 10, 12, 14, 16 depict the master side performance of Nisocia observer based approach, while Fig. 11, 13, 15, 17 show the slave side performance of Nisocia observer based approach. Specifically, Fig. i.a), Fig. i.c), and Fig. i.e) (i=10,12,14,16) show the trajectories of the error between desired operator force and estimated environment force in $Z_{m 1}$ direction, the error between actual and estimated operator force in $Z_{m 1}$ direction, and parameter- 1 of the master robot manipulator, while Fig. i.b), Fig. i.d), and Fig. i.f) (i=10,12,14,16) provide the trajectories of the error between desired operator force and estimated environment force in $Z_{m 2}$ direction, the error between actual and estimated operator force in $Z_{m 2}$ direction, and parameter-2 of the master robot manipulator. Meanwhile, Fig. j.a), Fig. j.c), and Fig. j.e) $(j=11,13,15,17)$ show the trajectories of the error between actual position of the master and slave robot manipulators in $Z_{s 1}$ direction, the error between actual and estimated environment force in $Z_{s 1}$ directions, and parameter-1 of the slave robot manipulator, while Fig. j.b), Fig. j.d), and Fig. j.f) $(j=11,13,15,17)$ provide the trajectories of the error between actual position of the master and slave robot manipulators in $Z_{s 2}$ direction, the error between actual and estimated environment force in $Z_{s 2}$ direction, and parameter-2 of the slave robot manipulator.

In Fig.10 and Fig. 11, simulation results are obtained in the absence of both robot parameter variations and measurement noises at both the master and slave sides of the teleoperation system. As observed in Fig.10, on the 
master end, the desired operator forces rendered in $Z_{m 1}$ and $Z_{m 2}$ directions are almost tracked by the environment forces rendered in $Z_{m 1}$ and $Z_{m 2}$ directions, and the operator force estimations are accurate. The trajectories of master robot parameter-1 and parameter-2 almost remain at 1.0 and 2.0, respectively. Similarly, on the slave end, as depicted in Fig. 11, positions of the master robot manipulator in $Z_{s 1}$ and $Z_{s 2}$ directions are accurately tracked by the positions of the slave robot manipulator in $Z_{s 1}$ and $Z_{s 2}$ directions respectively, and the environment force estimations are also accurate. The trajectories of slave robot parameter- 1 and parameter- 2 almost remain at 1.0 and 2.0, respectively as there is no parameter variation in the system.

Fig. 12 and Fig. 13 depict the performance of the EAOB based approach only with parameter variation: 20\% of the actual parameters at both the master and slave sides of the teleoperation system. In this situation, at the master side, as depicted in Fig. 12, the trajectories of force tracking in $Z_{m 1}$ and $Z_{m 2}$ directions deteriorate a little bit, the operator force estimation also becomes poor, and the trajectories of master robot parameter- 1 and parameter-2 do not converge from $(1.2,2.4)$ to $(1.0,2.0)$, respectively, and remain at $(1.2,2.4)$, as there is no parameter adaptation in the Nicosia observer based control scheme. Meanwhile, In Fig. 13, the trajectories of position tracking in $Z_{s 1}$ and $Z_{s 2}$ directions at the slave side are also accurate, but the environment force estimation starts to lose the accurate performance, and the trajectories of slave robot parameter- 1 and parameter2 do not converge from $(1.2,2.4)$ to $(1.0,2.0)$, respectively, and remain at $(1.2,2.4)$, the same as trajectories of master robot parameter-1 and parameter-2.

Fig. 14 and Fig. 15 provide the performance of the EAOB based approach when only measurement noise is considered in the teleoperation system. As shown in Fig. 14 and Fig. 15, it is easy to observe that when Gaussian noise $(N \sim(0,1.0 \mathrm{e}-5))$ is added on both the master and slave sides, the trajectories of force tracking in $Z_{m 1}$ and $Z_{m 2}$ directions at the master side become poor, while the trajectories of position tracking in $Z_{s 1}$ and $Z_{s 2}$ directions at the slave side remain accurate. The accuracy of the operator and environment force estimations also becomes unacceptable. The trajectories of the master and slave robot parameter- 1 and parameter- 2 adaptation remain at 1.0 and 2.0 , respectively.

In Fig. 16 and Fig. 17, the performance of the EAOB based approach with measurement noise: $N \sim(0,1.0 \mathrm{e}-5)$ and parameter variation: $20 \%$ of the actual parameters at both the master and slave sides of the teleoperation system is examined. From Fig. 16, it is easy to see that the trajectories of force tracking in $Z_{m 1}$ and $Z_{m 2}$ directions on the master end remain poor, and the operator force estimation is still very poor. Furthermore, the trajectories of master robot parameter-1 and parameter-2 do not converge from $(1.2,2.4)$ to $(1.0,2.0)$, 
respectively, and remain at $(1.2,2.4)$. Meanwhile, In Fig. 17, the trajectories of position tracking in $Z_{s 1}$ and $Z_{s 2}$ directions on the slave end remain accurate, but the environment force estimation is still unsatisfactory, and the trajectories of slave robot parameter- 1 and parameter-2 do not converge from $(1.2,2.4)$ to $(1.0,2.0)$, respectively, and remain at $(1.2,2.4)$.

\subsubsection{Comparison and analysis}

The diagrams illustrated in Fig. 2, 3 and Fig. 10, 11, clearly demonstrate that both EAOB and Nisocia observer based approach can effectively perform during force and position tracking as well as operator and environment force estimation when there are no parameter variation and measurement noise in the entire teleoperation system.

However, as depicted in Fig. 4, 5 and Fig. 12, 13, when parameter variations of $20 \%$ of the actual robot parameters are added to the initial estimated robot parameters at both the master and slave side of the teleoperation system, the performance of the EAOB based approach is nearly maintained. This is possible as the parameters of the robot are adapted in EAOB as seen in Fig. 4, 5. In contrast, the Nisocia observer based approach begins to lose its accurate performances on operator and environment force estimation. These results show that the EAOB based approach could achieve accurate force and position tracking as well as good force estimation in the presence of robot parameter variations, while the performance of Nisocia observer based approach becomes poor and poor as the parameter variation increases.

On the other hand, when measurement noise is considered in the teleoperation system, the EAOB based approach performs better compared to the Nisocia observer based approach in terms of force tracking, and especially force estimation at both the master and slave sides of the teleoperation, as seen in Fig. 6, 7 and Fig. 14, 15. When the Gaussian noise $(N \sim(0,1.0 \mathrm{e}-5))$ is added into both the master and slave sides of the system, the EAOB based approach can still obtain accurate force and position tracking and approximate force estimation, while the Nisocia Observer based approach can only attain approximate position tracking, but force tracking at master side and force estimation at both sides cannot be provided any more. These results indicate that the EAOB based approach can yield good performance for force and position tracking as well as force estimation in the presence of measurement noise, while the Nisocia observer based approach does not perform well in any respect, especially in terms of force tracking and force estimation.

Finally, the simulation results for the EAOB and Nisocia observer based approach with both measurement noise: $N \sim(0,1.0 \mathrm{e}-5)$ and parameter variation: $20 \%$ of actual parameters at the master and slave sides are depicted in 
Fig. 8, 9 and Fig. 16, 17. It is easy to see that the EAOB based approach still functions well in terms of force and position tracking, force estimation, and parameter adaptation, while the Nisocia observer based approach not only provides approximate position tracking, but also loses its performance on force tracking and estimation. These results imply the robustness of the EAOB based approach.

In summary, the analysis of simulation results presented in this Section well illustrate that the proposed EAOB based position-force control architecture for a teleoperation system can achieve accurate force and position tracking at the master and slave side respectively, as well as simultaneous operator and environment force estimation and parameter adaptation for nonlinear master and slave systems in the presence of robot parameter variations and measurement noises. The success of EAOB-based method is attributed to the following factors.

Firstly, as non-adaptive robot dynamic models use a fixed set of parameters that are no longer valid when the operating conditions change, the EAOB-based method has tunable inertial parameters that need to be adjusted in real time for optimal performance. It is relatively easy to select optimal values for entries in the process noise covariance matrix corresponding to the estimated states, and there are no significant performance losses for reasonable perturbations in tuned parameters. On the contrary, the Nicosia observer-based method does not update the inertial parameters in real time, and hence loses its ability estimating force. Meanwhile, since the EAOB deploys the theoretical concepts behind Kalman filter, it possesses good measurement and process noise rejection ability, which also makes the EAOB superior to the compared Nicosia observer.

\section{Conclusion}

This paper presents a novel Extended Active Observer (EAOB) based position-force control algorithm for a nonlinear bilateral teleoperation system that provides the benefits of a position-force architecture in terms of accurate force tracking. However, similar to a position-position architecture it does not require the use of force sensors. The results obtained show the ability of the Extended Active Observer (EAOB) based approach to simultaneously estimate position, velocity, inertial parameter and force based on measured position in the presence of measurement noise and parameter variation at both the master and slave sides of the teleoperation system. Through comparison of EAOB against a typical Nicosia observer applied to a typical 2 DOF nonlinear teleoperator, two major advantages of the proposed method were demonstrated.

Firstly, in EAOB, variation of the model parameters is compensated for in real time, ensuring accurate force estimation. In contrast, the Nisocia observer (or other force observers) was not able to accommodate robot 
parameter variations and did not produce accurate force estimation. This makes the proposed observer more reliable and effective in real world applications compared to others. Secondly, the proposed EAOB based approach also considers the effect of the measurement noise on system performance, and can effectively estimate force and track position in spite of measurement noise. On the contrary, the Nisocia observer (or other force observers) based approach proved to be incapable to function well in the presence of measurement noise.

While the performance of the EAOB based scheme has been explored through computer simulation, the experimental verification of the proposed approach is also outstanding. This will be the goal of the future work. Meanwhile, the proposed scheme mainly considers the inertial parameter variations and environment noise at both the master and slave side of the teleoperation system when estimating the external operator or environment force. In reality, however, a teleoperation system also suffers from communication delay between the master and the slave side ${ }^{16}$, which could result in poor force estimation, and even instability of the entire teleoperation system. Thus, a further development of the scheme for the teleoperation system is to study how EAOB can deal with communication delay issue.

\section{Reference}

1. D. A. Lawrence, “Stability and transparency in bilateral teleoperation,” IEEE Transactions on Robotics and Automation, 9(5), 624-637 (1993).

2. M. Tavakoli, A. Aziminejad, R. V. Patel, and M. Moallem, “Enhanced transparency in haptics-based master-slave systems,” American Control Conference, New York (2007) pp. 1455-1460.

3. W.H. Chen, D. J. Ballance, P. J. Gawthrop, and J. O’Reilly, “A Nonlinear Disturbance Observer for Robotic Manipulators,” IEEE Transactions On Industrial Electronics, 47( 4), 932-938 (2000).

4. J. M. Daly and D. W. L. Wang, "Bilateral Teleoperation Using Unknown Input Observers for Force Estimation,” American Control Conference, St. Louis, MO (2009) pp. 89-95.

5. H.S. Ahn, "Synchronization of Bilateral Teleoperation Systems using State and Force Observer," International Conference on Control, Automation and Systems, Gyeonggi-do, Korea (2010) pp.1362-1365.

6. S. Nicosia and P. Tomei, “Robot control by using only joint position measurements,” IEEE Transactions On Automatic Control, 35(9), 1058-1061 (1990)

7. R. Gourdeau and H. M. Schwartz, “Adaptive Control of Robotic Manipulators Using an Extended Kalman Filter,” Journal of Dynamic Systems, Measurement, and Control, 115(1), 203-208 (1993). 
8. J. Rosen, B. Hannaford, M. MacFarlane, and M. Sinanan, "Force controlled and teleoperated endoscopic grasper for minimally invasive surgery-experimental performance evaluation,” IEEE Transactions on Biomedical Engineering, 46(10), 1212-1221 (1999).

9. L. Ni and D. W. L. Wang, “A gain-switching control scheme for position-error-based bilateral teleoperation: Contact stability analysis and controller design,” International Journal of Robotics Research, 23(3), 255-274 (2004).

10. D. A. Lawrence, "Stability and transparency in bilateral teleoperation," IEEE Transactions on Robotics and Automation, 9(5), 624-637 (1993).

11. R. Cortesão, “On Kalman Active Observers,” Journal of Intelligent Robot Systems, 48(2), 131-155 (2007).

12. R. Cortesão, R. Koeppe, U. Nunes, and G. Hirzinger, “Data fusion for robotic assembly tasks based on human skills,” IEEE Transactions on Robotics, 20(6), 941-952 (2004).

13. G. Richard, Adaptive control of robotic manipulators (Ph.D. thesis, Carleton University, 1990).

14. R.E. Kalman and R.S. Bucy, “New results in linear filtering and prediction theory,” ASME journal of Basic engineering, 83(1), 95-108 (1961).

15. L. Sciavicco and B. Siciliano, Modeling and Control of Robot Manipulators (McGraw-Hill, New York, 1996).

16. P. F. Hokayem, and M.W. Spong, “Bilateral teleoperation: An historical survey,” Automatica, 42(12), $2035-2057$ (2006). 